\title{
Proteinase 3 on apoptotic cells disrupts immune silencing in autoimmune vasculitis
}

\author{
Arnaud Millet, ${ }^{1,2,3,4}$ Katherine R. Martin, ${ }^{1,2,3,4}$ Francis Bonnefoy, ${ }^{5}$ Philippe Saas, ${ }^{5}$ Julie Mocek, ${ }^{1,2,3,4}$ Manal Alkan, $, 2,2,3,4$ \\ Benjamin Terrier, ${ }^{1,2,3,4,6}$ Anja Kerstein, ${ }^{7}$ Nicola Tamassia, ${ }^{8}$ Senthil Kumaran Satyanarayanan, ${ }^{9}$ Amiram Ariel, ${ }^{9}$ \\ Jean-Antoine Ribeil, ${ }^{3,10}$ Loïc Guillevin, ${ }^{6}$ Marco A. Cassatella, ${ }^{8}$ Antje Mueller, ${ }^{7}$ Nathalie Thieblemont, ${ }^{1,2,3,4}$ Peter Lamprecht, ${ }^{7}$ \\ Luc Mouthon, ${ }^{1,2,3,4,6}$ Sylvain Perruche, ${ }^{5}$ and Véronique Witko-Sarsat ${ }^{1,2,3,4}$ \\ ${ }^{1}$ INSERM U1016 Cochin Institute, Paris, France. ${ }^{2}$ CNRS UMR 8104, Paris, France. ${ }^{3}$ Université Paris-Descartes, Paris, France. ${ }^{4}$ Center of Excellence, LabEx INFLAMEX, Paris, France. ${ }^{5}$ INSERM UMR 1098, EFS BFC, \\ University Bourgogne Franche-Comté, LabEx LipSTIC, Besançon, France. ${ }^{6}$ Department of Internal Medicine, Cochin Hospital, Centre de Référence pour les Maladies Auto-immunes Rares, Assistance \\ Publique-Hôpitaux de Paris, Paris, France. 'Department of Rheumatology, University of Lübeck and Vasculitis Center UKSH, Lübeck, Germany. ${ }^{8}$ Department of Medicine, Faculty of \\ Medicine, Verona, Italy. ${ }^{~}$ Department of Biology, Faculty of Natural Sciences, University of Haifa, Haifa, Israel. ${ }^{10}$ Department of Biotherapy, Necker Hospital, Paris, France.
}

\begin{abstract}
Granulomatosis with polyangitis (GPA) is a systemic necrotizing vasculitis that is associated with granulomatous inflammation and the presence of anti-neutrophil cytoplasmic antibodies (ANCAs) directed against proteinase 3 (PR3). We previously determined that PR3 on the surface of apoptotic neutrophils interferes with induction of antiinflammatory mechanisms following phagocytosis of these cells by macrophages. Here, we demonstrate that enzymatically active membrane-associated PR3 on apoptotic cells triggered secretion of inflammatory cytokines, including granulocyte CSF (G-CSF) and chemokines. This response required the IL-1R1/MyD88 signaling pathway and was dependent on the synthesis of NO, as macrophages from animals lacking these pathways did not exhibit a PR3-associated proinflammatory response. The PR3-induced microenvironment facilitated recruitment of inflammatory cells, such as macrophages, plasmacytoid DCs (pDCs), and neutrophils, which were observed in close proximity within granulomatous lesions in the lungs of GPA patients. In different murine models of apoptotic cell injection, the PR3-induced microenvironment instructed pDC-driven Th9/Th2 cell generation. Concomitant injection of anti-PR3 ANCAs with PR3-expressing apoptotic cells induced a Th17 response, revealing a GPA-specific mechanism of immune polarization. Accordingly, circulating CD4+ $T$ cells from GPA patients had a skewed distribution of Th9/Th2/Th17. These results reveal that PR3 disrupts immune silencing associated with clearance of apoptotic neutrophils and provide insight into how PR3 and PR3-targeting ANCAs promote GPA pathophysiology.
\end{abstract}

\section{Introduction}

Granulomatosis with polyangiitis (GPA) is a systemic autoimmune disease characterized by granulomatous inflammation and necrotizing vasculitis (1). GPA is associated with anti-neutrophil cytoplasmic antibodies (ANCAs) directed against the neutrophil- and monocyte-derived serine protease, proteinase 3 (PR3). A GWAS identified a genetic association between a single nucleotide polymorphism in the PR3 gene and anti-PR3 ANCA (2). Interestingly, there was no genetic association between anti-myeloperoxidase (anti-MPO) ANCA and their target MPO, another neutrophil protein and the main target of ANCA in microscopic polyangiitis (MPA). This genetic analysis strongly suggests that PR3 is a central player in the autoimmune dysregulation associated with GPA. PR3, also known as myeloblastin $(3,4)$, regulates granulocyte differentiation in promyelocytes via the cleavage of cyclin-dependent kinase inhibitor p21/waf1 (5). PR3 is a granulocyte CSFresponsive (G-CSF-responsive) gene, which can be reexpressed in vivo during inflammation (6). Moreover, compared with its

Authorship note: Arnaud Millet and Katherine R. Martin contributed equally to this work. Conflict of interest: The authors have declared that no conflict of interest exists. Submitted: July 23, 2014; Accepted: August 27, 2015.

Reference information: / Clin Invest. 2015;125(11):4107-4121. doi:10.1172/JCI78182. homologue neutrophil elastase, PR3 possesses structural and functional features that may contribute to its involvement in autoimmune vasculitis (7). For example, while this protein is mainly stored within azurophilic granules of mature neutrophils, PR3 can also be expressed at the plasma membrane under basal conditions (8). In fact, a higher percentage of neutrophils express membrane PR3 in GPA patients compared with healthy controls (9). This constitutive PR3 membrane expression may favor anti-PR3 ANCA binding and subsequent neutrophil activation, which in turn could promote inflammation $(10,11)$. Additionally, dysregulation of $P R 3$ mRNA expression and epigenetic control has been documented in neutrophils from GPA patients $(12,13)$. We have also demonstrated that PR3 is expressed on the surface of apoptotic neutrophils $(14,15)$, where it can interfere with the phagocytosis of apoptotic cells by macrophages. This process, known as efferocytosis (16-18), could in turn lead to nonresolving inflammation $(19,20)$. These experiments revealed an association between PR3 and calreticulin (21), an "eat-me signal" expressed at the membrane of apoptotic neutrophils that is implicated in the recognition of dying cells by macrophages (22). These findings lead to the hypothesis that PR3 expressed on the surface of apoptotic neutrophils impairs the resolution of inflammation not only by delaying their phagocytosis but also by shaping macrophage polarization. This pro- 
cess could in turn affect subsequent immune silencing and may potentially involve DCs and T lymphocytes. DCs play a pivotal role in immune silencing, notably through their ability to migrate from the periphery into the thymus, transporting peripheral antigens captured from apoptotic cells and promoting the generation of Tregs $(23,24)$. DCs have been identified in inflammatory lesions of GPA patients $(25,26)$. Interestingly, plasmacytoid DCs (pDCs), which favor Treg production, are involved in this process (27). These cells can induce immune tolerance in response to the macrophage microenvironment generated following exposure to apoptotic cells (28). The aim of the present study was to evaluate the consequences of PR3 expression on apoptotic cells on different cellular players implicated in the immune crosstalk following efferocytosis. To achieve this, we examined the effect of PR3 expression at the surface of apoptotic cells on macrophage, pDC, and $\mathrm{T}$ cell functions in vitro and in vivo.

\section{Results}

Membrane expression of PR3 increased during neutrophil apoptosis in GPA patients. Spontaneous apoptosis of neutrophils within whole blood from GPA patients was diminished compared with that of age- and sex-matched healthy controls, where the percentage of annexin $\mathrm{V}^{+}$cells was $42 \% \pm 6 \%$ in healthy controls $(n=8)$ versus $22 \% \pm 8 \%$ in GPA patients $(n=10)$ (data expressed as mean \pm SEM, $P<0.05$, Mann-Whitney $U$ test); this observation was in line with previously published results (29). In accordance with previous findings showing that PR3 was expressed at the plasma membrane of apoptotic neutrophils (14), significantly higher PR3 membrane expression was observed on apoptotic neutrophils isolated from GPA patients compared with healthy controls (Figure 1A). Further, there was no increase in the expression of other membrane-bound proteins, such as CD11b (Figure 1B). As PR3 membrane expression on neutrophils can increase following TNF- $\alpha$ treatment, the relationship between membrane-bound PR3 in basal and TNF- $\alpha-$ activated neutrophils was analyzed in healthy controls and GPA patients. As expected, basal and TNF- $\alpha$-induced PR3 membrane expression were tightly correlated (Figure 1C). In contrast, PR3 membrane expression induced during apoptosis did not correlate to basal membrane expression (Figure 1D), strongly suggesting that PR3 membrane expression after apoptosis occurs independently of degranulation. This observation led to the hypothesis that there are different pools of PR3 within neutrophils that can be expressed at the plasma membrane. Using confocal microscopy, double immunolabeling of PR3 and cleaved caspase-3 showed that both proteins colocalized in neutrophils within granulomatous lesions from the nasal mucosa of GPA patients (Figure 1E).

Membrane-associated PR3 expressed on apoptotic cells triggered a proinflammatory response in macrophages in vivo. To study the effect of PR3 expressed on apoptotic cells, rat basophilic leukemia (RBL) cells stably transfected with human PR3, as described previously $(14,21)$, were used to mimic neutrophils. As in human neutrophils, membrane expression of PR3 can be induced by either degranulation or apoptosis (30). Further, as RBL cells do not express other neutrophil serine proteases, PR3-mediated processes can be studied exclusively. Injection i.p. of apoptotic cells expressing PR3 in mice induced a proinflammatory response, as demonstrated by increased concentrations of IL-6, TNF- $\alpha$, CXCL1 (KC), IL-12p70, monocyte chemoattracting protein-1 (MCP-1), IL-1 $\beta$, G-CSF, and IL-2 in the peritoneal lavage collected from mice injected with PR3expressing cells compared with controls (Figure 2A). Polarization of macrophages was also examined 24 hours after injection of apoptotic cells, and while no change in the expression of CD11b, TLR4, MHC II, CD11c, CD23, or CD16/CD32 was observed on F4/80+ macrophages, apoptotic cells expressing PR3 induced a decrease in the expression of CD206, a known M2 marker (Figure 2B). To determine whether the serine protease activity of PR3 was required for this proinflammatory response, an inactive PR3 mutant (PR3/ S203A) containing a mutation of the serine 203 from the catalytic triad as previously described (30) was used (Supplemental Figure 1; supplemental material available online with this article; doi:10.1172/JCI78182DS1). A significant decrease in MCP-1 was observed in the peritoneal lavage from mice injected with apoptotic cells expressing PR3/S203A compared with those expressing enzymatically active PR3, demonstrating that the protease activity was essential for its proinflammatory effect (Figure 2C).

To examine the effects of apoptotic cells expressing PR3 on inflammatory macrophages, a murine thioglycolate-induced peritonitis model was used. Injection i.p. of apoptotic control or PR3expressing cells was performed 72 hours after the induction of peritonitis and the lavages analyzed 24 hours later. As expected, injection of apoptotic control cells resulted in a reduction in proinflammatory chemokines (macrophage inflammatory protein- $1 \alpha$ [MIP-1 $\alpha$ ], MIP-1 $\beta$, MCP-1, and RANTES) compared with vehicle alone (Figure 2D). In contrast, injection of apoptotic cells expressing PR3 significantly impaired antiinflammatory effects, as illustrated by increased MIP- $1 \alpha$ and MIP- $1 \beta$ compared with control cells. Accordingly, the number of macrophages recruited to the peritoneal cavity was significantly reduced in mice injected with apoptotic cells $\left(0.65 \pm 0.14 \times 10^{6}\right.$ and $0.83 \pm 0.09 \times 10^{6}$ for control and PR3 cells, respectively) compared with those injected with thioglycolate alone $\left(1.41 \pm 0.17 \times 10^{6}\right.$, data expressed as mean \pm SEM, $P<0.05$, Mann-Whitney $U$ test). This observation indicated that injection of apoptotic cells expressing PR3 did not potentiate the influx of macrophages into the peritoneal cavity, but rather they switched the recruited cells toward a proinflammatory profile.

$P R 3$ membrane anchorage on apoptotic cells was required to induce a proinflammatory response in macrophages. To investigate the mechanisms involved in the proinflammatory response induced by PR3, thioglycolate-elicited macrophages were cultured in vitro and exposed to apoptotic cells expressing either no PR3 (control), WT PR3, or a membrane anchorage mutant (PR3/4H4A) that cannot be expressed at the plasma membrane during apoptosis (31). Under these conditions, no difference in the phagocytosis index of all apoptotic cell types was observed (data not shown). Analysis of macrophage supernatants indicated that apoptotic cells expressing PR3 not only induced the secretion of proinflammatory cytokines, such as IL-6, IL-1 $\beta$, TNF- $\alpha$, and IL-12p70, but also that of chemokines implicated in the recruitment of PR3-expressing cells (neutrophils and monocytes), including MIP-1 $\alpha$, MCP-1, RANTES, and $\mathrm{KC}$ (Figure 3A). This proinflammatory signature was not observed for control apoptotic cells or apoptotic cells expressing PR3/4H4A. Of note, the phagocytosis of apoptotic cells was associated with secretion of TGF- $\beta 1$, but this secretion did not significantly vary when PR3 was expressed on the membrane (435.5 \pm 


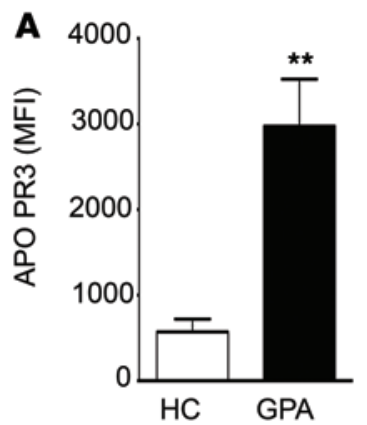

B

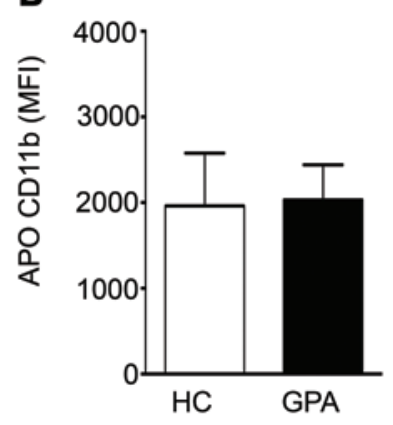

C

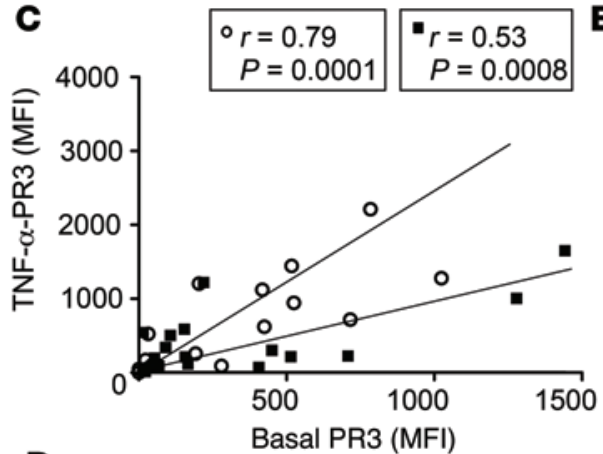

D

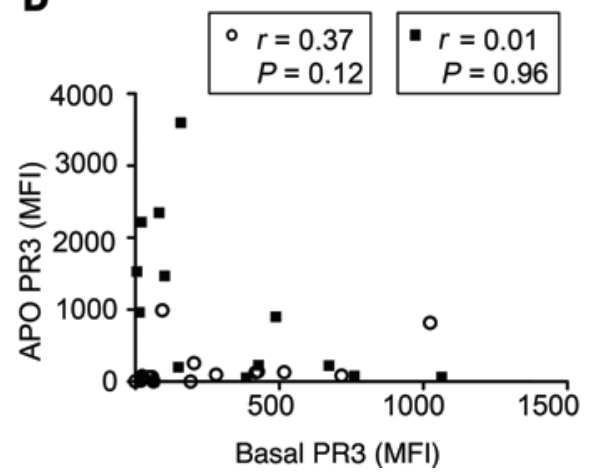

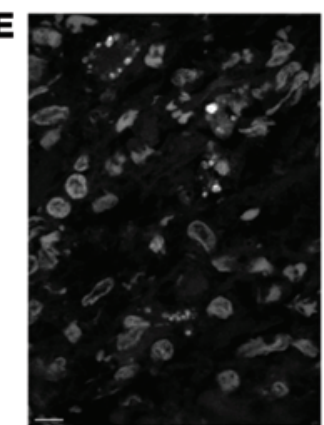
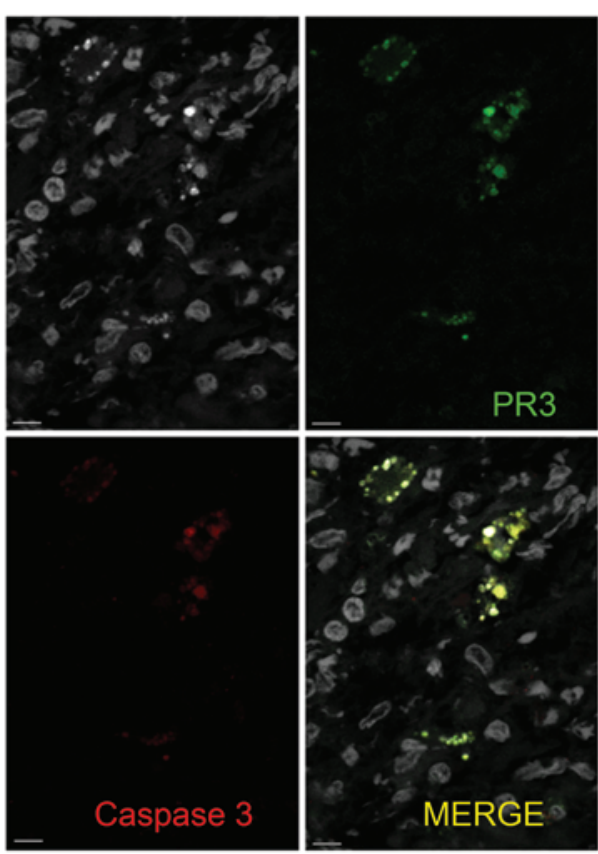

Figure 1. PR3 membrane expression on apoptotic neutrophils was increased in GPA. (A and B) Membrane expression of PR3 and CD11b on apoptotic neutrophils (CD15+Annexin $\left.\mathrm{V}^{+} 7 \mathrm{AAD}{ }^{-}\right)$from healthy controls $(\mathrm{HC})(n=8)$ and GPA patients $(n=10)$ was assessed in whole blood maintained at $37^{\circ} \mathrm{C}$ for 20 hours. Values are presented as mean \pm SEM. ${ }^{*} P<0.01$. (C) PR3 expression on unstimulated neutrophils (basal) correlated with PR3 expression on TNF- $\alpha-$ stimulated neutrophils. (D) No correlation in membrane expression of PR3 (mean fluorescence intensity [MFI]) on basal and apoptotic neutrophils was observed. Data are presented for $n=19$ healthy controls (white circles) and $n=15$ GPA (black squares) with a mean age of $47.6 \pm 3.2$ versus $51.7 \pm 4.4$ years, respectively, and with a similar sex ratio, provided in Supplemental Table 1. (E) Within granulomatous inflammation in GPA, PR3+ (green, upper right panel) and cleaved caspase $3^{+}$(red, lower left panel) cells detected by immunofluorescence analyses using confocal microscopy colocalized within the same cell (merge, lower right panel). Nuclei visualized using DAPI are depicted in gray (upper left panel). Scale bars: $5 \mu$ m. Significant differences between groups were determined by Mann-Whitney $U$ test (A and $\mathbf{B})$, and correlations were assessed using Pearson's tests (C and $\mathbf{D})$.

49.7 vs. $389.9 \pm 42.7 \mathrm{pg} / \mathrm{ml}$ for controls versus PR3, respectively, data expressed as mean \pm SEM, $n=10$ per group). Finally, the proinflammatory response elicited by PR3 was also demonstrated by the strong expression of inducible NOS (iNOS) observed in macrophages following the phagocytosis of apoptotic cells expressing membrane PR3, while no induction was observed after phagocytosis of control or PR3/4H4A cells (Figure 3B). Taken together, these results demonstrate that the proinflammatory signature in macrophages following the phagocytosis of apoptotic cells expressing PR3 was strictly dependent on the presence of this protein at the plasma membrane during apoptosis.

PR3 impaired the antiinflammatory reprogramming of macrophages following ingestion of apoptotic cells. Efferocytosis of apoptotic cells by macrophages downregulated the expression of proinflammatory cytokines following treatment with LPS, demonstrating successful macrophage reprogramming $(32,33)$. Indeed, both apoptotic control and PR3/4H4A-expressing cells downregulated the production of IL-6, granulocyte-macrophage CSF (GM-CSF), IL-12p70, and MIP-1 $\alpha$ compared with macrophages stimulated with LPS alone (Figure $3 \mathrm{C}$ ). In contrast, apoptotic cells expressing membrane PR3 impaired macrophage reprogramming, as demonstrated by increased secretions of the above cytokines compared with macrophages ingesting apoptotic control or PR3/4H4A cells (Figure 3C).
Membrane-associated PR3 on apoptotic cells acts as a danger signal through the IL-1R1/MyD88 signaling pathway in a NO-dependent manner. Macrophages from $M y d 88$-deficient mice were isolated following thioglycolate-induced peritonitis and exposed to either apoptotic control or PR3-expressing cells for 24 hours in vitro. Notably, an increased secretion of MCP-1 was observed in macrophages from

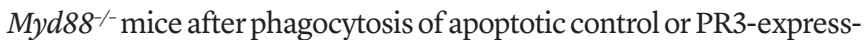
ing cells compared with macrophages from WT mice. Nonetheless, no difference in MCP-1 secretion was observed between apoptotic control and PR3-expressing cells, strongly suggesting that the MyD88 pathway was involved in the proinflammatory response elicited by PR3 (Figure 4). To identify the receptor or receptors involved, the above experiments were performed using macrophages isolated from Tlr2 $2^{--}$, Tlr4 ${ }^{-/}$, or Illr1 $1^{-/}$mice (Figure 4). A PR3-induced inflammatory response, as evidenced by increased MCP-1 production following phagocytosis of apoptotic PR3-expressing cells and not control apoptotic cells, was observed in $\mathrm{Tlr} 2^{-/-}$or $\mathrm{Tlr} 4^{-/-}$macrophages. In contrast, no increase in MCP-1 secretion following phagocytosis of apoptotic cells expressing PR3 was observed in macrophages from $\mathrm{Illr}^{-/-}$mice, indicating that the IL-1 receptor was required for the proinflammatory response. Additionally, no induction of MCP-1 was detected in macrophages after phagocytosis of apoptotic cells expressing PR3 in Nos $2^{-/-}$mice, strongly suggesting that NO production was a key mediator in the proinflammatory pathway induced by PR3. 
A
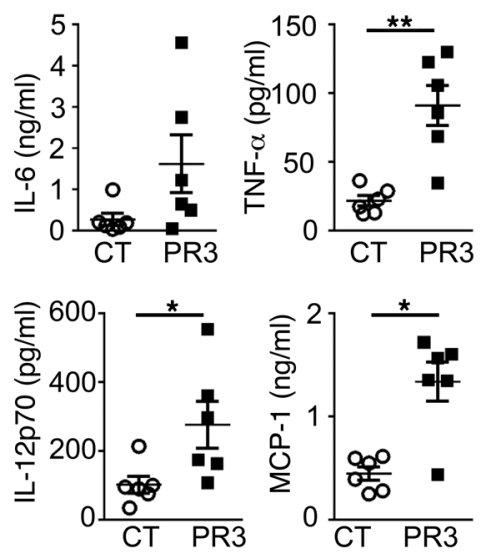

B

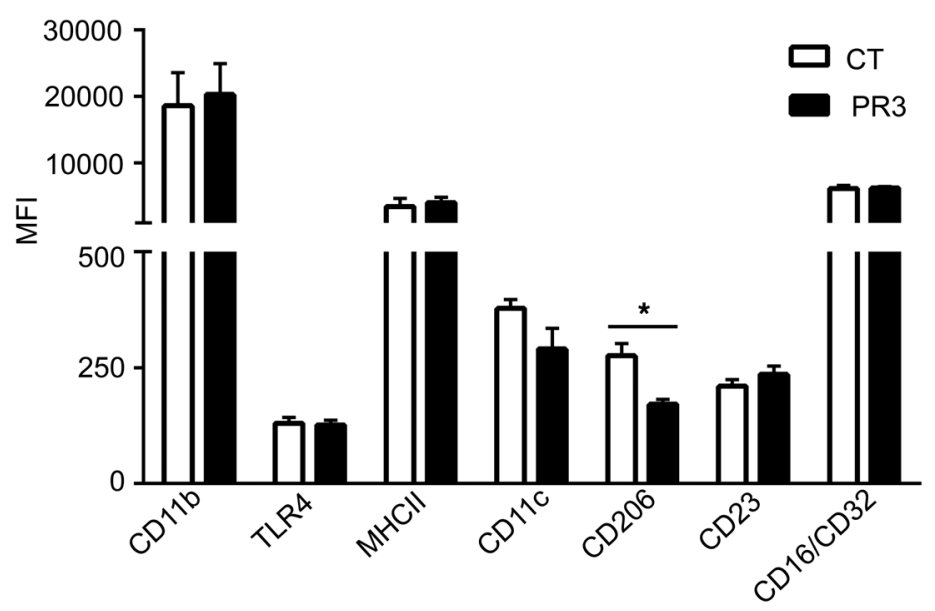

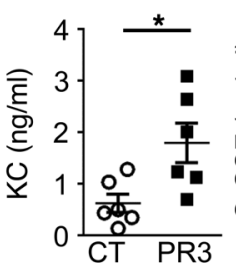
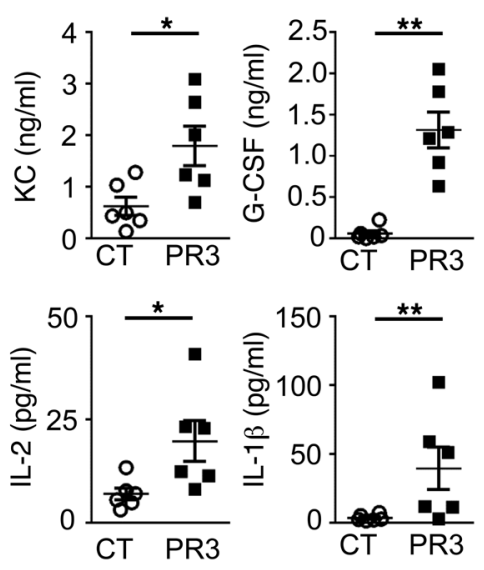

C

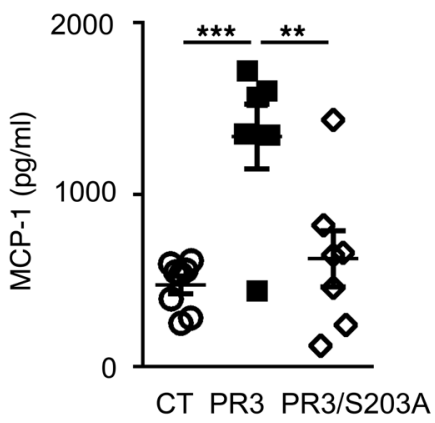

D
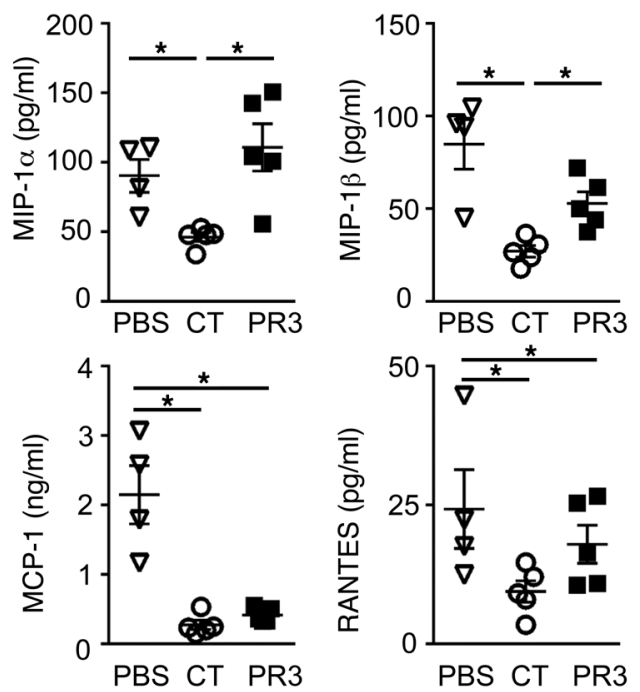

Figure 2. Membrane expression of PR3 on apoptotic cells triggered a proinflammatory response in vivo that required serine protease activity. (A) Apoptotic control (CT) (white circles) or PR3-expressing (black squares) cells were injected i.p. and peritoneal lavage fluid collected after 2 hours. IL-6, TNF- $\alpha$, KC, G-CSF, IL-12p70, MCP-1, IL-2, and IL-1 $\beta$ were assessed using the proinflammatory mouse cytokine assay ( $n=5$ mice per group, each cytokine measured in triplicate). (B) Using flow cytometry, polarization of $\mathrm{F} 4 / 80^{+}$macrophages was examined by assessing expression of various cell-surface markers, including CD11b, TLR4, MHCII, CD11c, CD206, CD23, and CD16/CD32 ( $n=4$ mice per group, each marker measured in duplicate). (C) Apoptotic control ( $n=8)$, PR3- $(n=6)$, or PR3/S203A-expressing cells $(n=8$, white diamonds) were injected i.p. in mice for 2 hours as in $\mathbf{A}$ and MCP-1 measured in duplicates in the peritoneal lavage by ELISA. (D) PBS (white triangles), apoptotic control, or PR3-expressing cells were injected i.p. 72 hours after peritonitis was induced with thioglycolate. The peritoneal lavage fluid was collected after a further 24 hours and concentrations of MIP-1 $\alpha$, MIP-1 $\beta$, MCP-1, and RANTES determined ( $n=5$ mice per group, each cytokine measured in triplicate). Data are presented as mean $\pm S E M .{ }^{*} P<0.05 ;{ }^{* *} P<0.01 ;{ }^{* * *} P<0.001$. Significant differences between groups were determined by multicomparison ANOVA (A-C) or Mann-Whitney $U$ test (D).

The PR3-induced microenvironment instructs pDCs to drive Th2/Th9 cell generation in vitro. Prior to coculture with naive CD4 ${ }^{+}$ T cells, pDCs isolated from BM of WT mice were exposed to the supernatant produced by macrophagesin the presence of apoptotic control or PR3-expressing cells. After 24 hours, pDCs were extensively washed with PBS and cocultured with naive $\mathrm{CD} 25^{-} \mathrm{CD} 4^{+}$ $\mathrm{T}$ cells from the thymus of Rag1/- OTII mice in the presence of $\mathrm{OVA}_{322-339}$ peptide for 4 days and $\mathrm{T}$ cell polarization evaluated by flow cytometry using CD25 and FOXP3 as well as IFN- $\gamma$ and IL-17A intracellular staining (Figure 5A). In the absence of apoptotic cells, Th1 $\left(\mathrm{IFN}-\gamma^{+} \mathrm{CD} 4^{+}\right)$polarization was predominant. Treatment with the supernatants produced by macrophages following the phagocytosis of apoptotic control cells led to a significant increase in the proportion of Tregs $\left(\mathrm{CD} 25^{+} \mathrm{FOXP}{ }^{+} \mathrm{CD} 4^{+}\right)$and Th17 cells $\left(\mathrm{IL}-17 \mathrm{~A}^{+} \mathrm{CD} 4^{+}\right)$and a downregulation of Th1 polarization.
In contrast, the supernatants produced by macrophages following phagocytosis of apoptotic cells expressing PR3 failed to induce Tregs or Th17 cells with a trend toward increased production of Th1 cells (Figure 5B). Analysis of cytokine secretion in the supernatant following coculture of pDCs with naive $\mathrm{CD} 4^{+} \mathrm{T}$ cells demonstrated a skewing toward Th2 (IL-4, IL-5) and Th9 (IL-9) profiles in the PR3-induced microenvironment (Figure 5C). Further, secreted levels of IL-17A and IFN- $\gamma$ in the supernatant confirmed results obtained using intracellular staining (Figure 5B). Cytokines secreted from pDCs alone following exposure to the same supernatants used to generate results in Figure 5B produced negligible levels of IL-4, IL-5, IL-9, IL-17A, or IFN- $\gamma$, supporting the assumption that these cytokines were secreted by $\mathrm{T}$ cells.

Injection i.v. of PR3-expressing apoptotic cells in mice polarized $T$ helper cells. To evaluate the ability of apoptotic cells expressing 

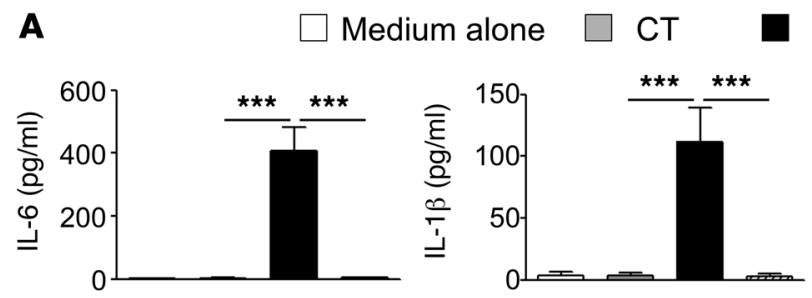

PR3

PR3/4H4A
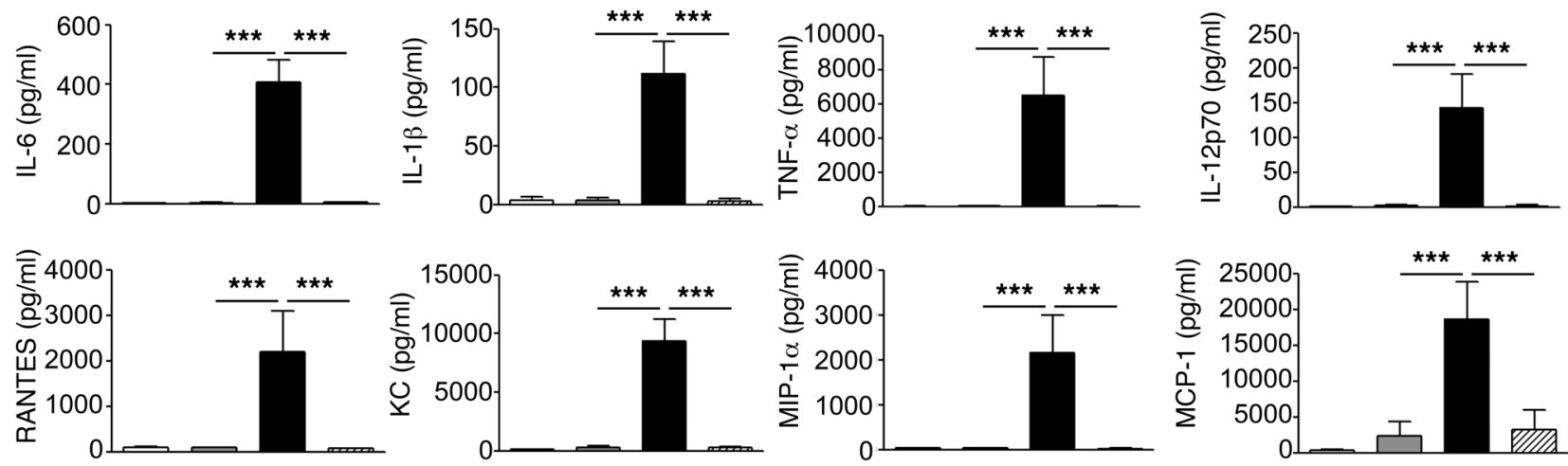

B
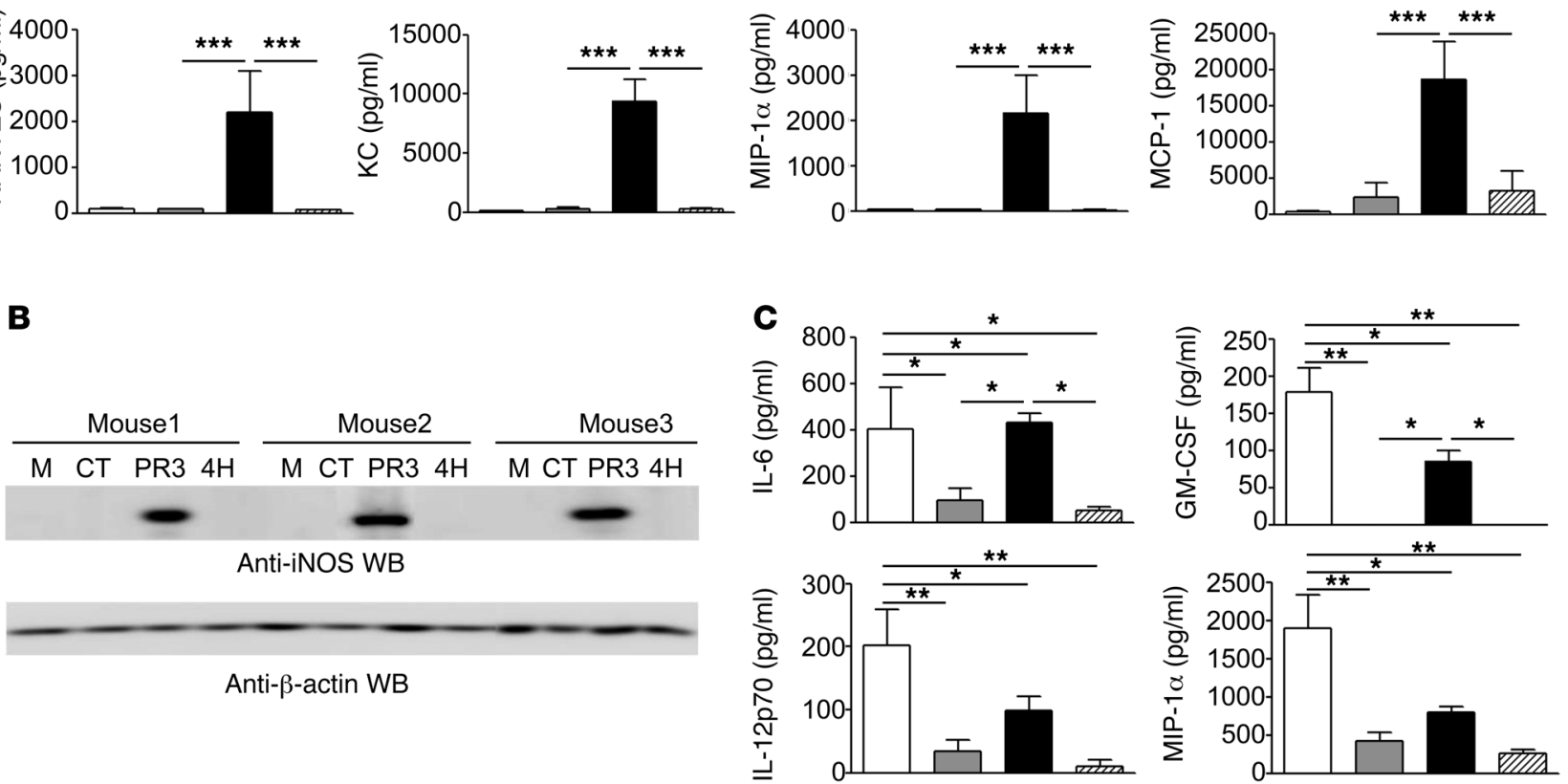

Figure 3. Membrane expression of PR3 on apoptotic cells induced a proinflammatory response in macrophages and impaired their antiinflammatory reprogramming following efferocytosis in vitro. (A) Thioglycolate-elicited macrophages were cultured for 24 hours in vitro with medium alone, with control apoptotic cells or with apoptotic cells expressing either PR3 or PR3/4H4A. Cytokine (IL-6, IL-1 $\beta$, TNF- $\alpha$, IL-12p70) and chemokine (RANTES, KC, MIP-1 $\alpha$, and MCP-1) production were assessed using a Luminex assay ( $n=4$ mice per group, each cytokine measured in triplicate; mean \pm SEM). (B) iNOS expression in macrophages was evaluated using Western blot analysis $(135 \mathrm{kDa})$, and $\beta$-actin was used as a loading control. Results represent 3 independent experiments. (C) Macrophages previously exposed to medium alone, apoptotic control cells, or PR3 or PR3/4H4A cells were stimulated with LPS (10 ng/ml) for 24 hours. Culture media was assessed for IL-6, GM-CSF, IL-12p70, and MIP-1 $\alpha$ using a Luminex assay. Values are represented as mean \pm SEM, $n=4$ mice per group, each cytokine measured in triplicate. ${ }^{*} P<0.05$; ${ }^{* *} P<0.01$; ${ }^{* *} P<0.001$. Significant differences between groups were determined by multicomparison ANOVA (A and $\mathbf{C})$.

PR3 to polarize $\mathrm{CD}^{+} \mathrm{T}$ cells in vivo, mice were injected with apoptotic cells i.v. pDCs exposed in vivo to the apoptotic cell-induced microenvironment were isolated from the spleen, a major site of apoptotic cell clearance (34-36), and these pDCs were subsequently cocultured with naive $\mathrm{CD} 25^{+} \mathrm{CD} 4^{+} \mathrm{T}$ cells from OTII $\mathrm{Rag1}^{-/-}$transgenic mice in the presence of $\mathrm{OVA}_{323-339}$ peptide for 4 days. Only pDCs exposed to apoptotic cells expressing PR3 in vivo induced a strong increase in Th9 cells compared with pDCs exposed to apoptotic control or PR3/4H4A cells (Figure 6A).pDCs exposed in vivo to apoptotic cells expressing PR3 induced Th1 and Th2 polarization with no Th17 cells and few Tregs observed, while the opposite $\mathrm{T}$ cell distribution was obtained when $\mathrm{pDC}$ s were preexposed to apoptotic control or PR3/4H4A cells (Figure 6A). Notably, the concomitant injection of both apoptotic cells expressing PR3 and anti-PR3 ANCA induced Th17 cell polarization (Figure $6 \mathrm{~B})$. Taken together, this in vivo data provide clear evidence that the autoantigen PR3 expressed on apoptotic cells triggered a Th9/ Th2/Th1 polarization and this response was further modulated by the presence of anti-PR3 ANCA, resulting in a modification of pDC activation leading to a sustained Th17 response.
To translate these results into a clinically relevant setting, peripheral blood mononuclear cells (PBMCs) were isolated from GPA patients with active disease and compared with those of healthy controls (Supplemental Table 1). Using intracellular staining of IFN- $\gamma$, IL-17A, IL-4, and IL-9 performed on $\mathrm{CD}^{+} \mathrm{CD}^{+} \mathrm{T}$ cells, GPA patients displayed a skewed Th9 cell distribution and a trend toward a skewed Th17 cell distribution compared with healthy controls (Figure 7, A and B). This was accompanied by an increase in typical Th2 (IL-4, IL-5) and Th9 (IL-9) cytokines, including increased levels of IL-17A (Figure 7C) as well as cytokines from the IL- 1 family, IL-1 $\beta$ and IL-1 receptor antagonist (IL-1RA) (Figure 7D), in the sera of GPA patients compared with healthy controls.

pDCs are located within close proximity to macrophages and apoptotic neutrophils expressing PR3 within granulomatous inflammation. In addition to granulomatous inflammation featuring neutrophils, macrophages, and $\mathrm{T}$ lymphocytes (including Tregs) in close proximity (37), IFN- $\alpha^{+} \mathrm{CD} 123^{+}$cells representing pDCs were also identified (Figure $8 \mathrm{~A}$ ). Some IFN- $\alpha^{+} \mathrm{CD} 123^{+}$cells were located at the edge of neutrophilic microabscesses in lung tissue 


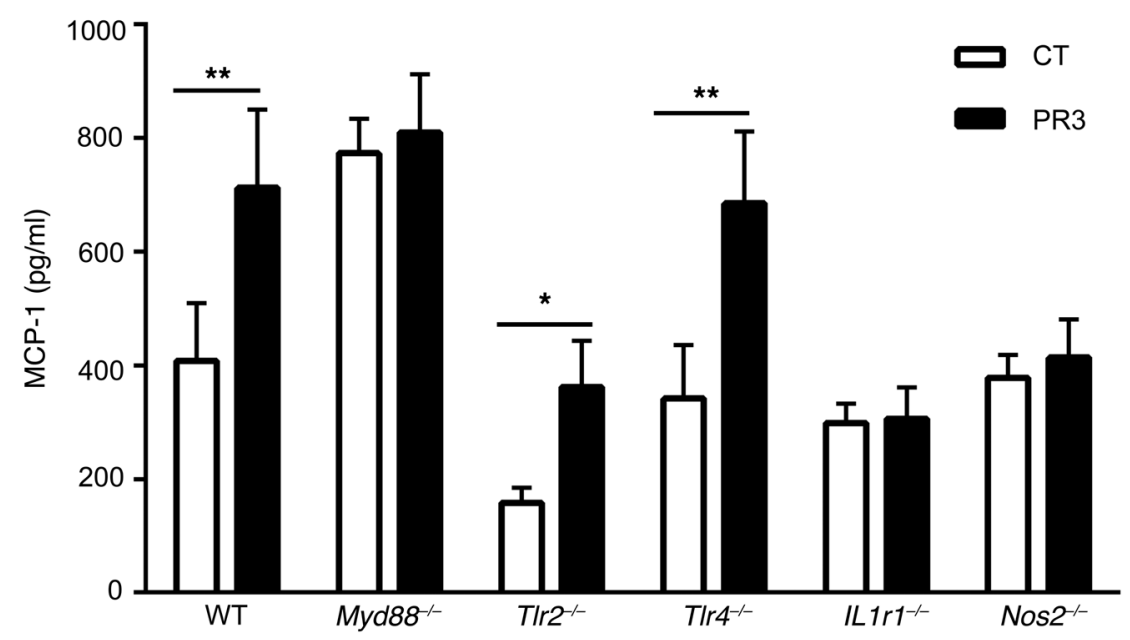

Figure 4. The proinflammatory response induced by PR3 on apoptotic cells was dependent on the IL-1R1/MyD88 signaling pathway and mediated by NO. Thioglycolate-elicited macrophages from C57BL/6 $(n=11)$, Myd88 $^{-/-}(n=12)$, Tlr2 $2^{-/-}(n=8)$,

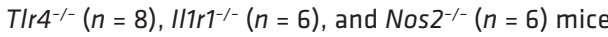
were cultured for 24 hours with apoptotic control or PR3-expressing cells and MCP-1 secretion was assessed in duplicate by ELISA. Values are shown as mean \pm SEM. ${ }^{*} P<0.05 ;{ }^{*} P<0.01$. Significant differences between groups were determined by the Mann-Whitney $U$ test. from GPA patients (Figure 8B). Serial staining of neutrophils (PR3 ${ }^{+}$ and $\mathrm{LL} 37^{+}$cells), macrophages $\left(\mathrm{CD} 68^{+}\right)$, and IFN- $\alpha^{+}$cells in nasal mucosa of GPA patients demonstrated a spatial interaction among all cell types (Figure 8, C-E). CD123 ${ }^{+}$cells were also located within close proximity of other immune cells, including neutrophils, that appear pyknotic (ref. 38 and Supplemental Figure 2A) or perhaps had undergone karyorrhexis (1). Some $\mathrm{CD} 123^{+}$cells also displayed nuclear staining, and this likely represented histiocytes, which have previously been described in granulomatous inflammation (39). Taken together, our data strongly suggest that within granulomatous inflammation, pDCs are located and presumably interact with macrophages and apoptotic neutrophils expressing PR3 (Supplemental Figure 2B).

PR3-impaired efferocytosis induced a G-CSF-driven autoamplification loop. G-CSF is an essential regulator of neutrophil production, increases proliferation of neutrophil progenitors, and promotes their release from BM (40). Apoptotic cells expressing PR3 strongly induced secretion of G-CSF by macrophages compared with control cells both in vivo (Figure 2A) and in vitro (Figure 9A). Likewise, CD4 ${ }^{+}$ T cells from OTII Rag1 ${ }^{-/-}$mice cocultured with pDCs preexposed to a PR3-induced microenvironment generated significantly higher levels of G-CSF compared with the control microenvironment (Figure 9B). Additionally, sera from GPA patients exhibited a higher concentration of G-CSF compared with those from healthy controls (Figure 9C). Notably, increased intracellular PR3 was detected by flow cytometry in neutrophils from GPA patients compared with controls (Figure 9D). Increased PR3 content was also detected using Western blot analysis in neutrophils from GPA patients compared with healthy controls (Figure 9E). This finding is in accordance with previous reports that PR3 is a G-CSF-responsive gene (41). This higher PR3 content in neutrophils from GPA patients was comparable to that observed in neutrophils isolated from G-CSF-treated healthy controls, a treatment used to improve collection of hematopoietic stem cells, indicating that PR3 was indeed induced by G-CSF in vivo
A

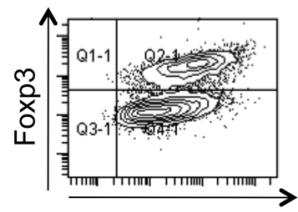

CD25

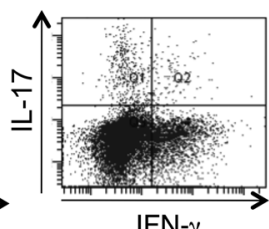

IFN- $\gamma$

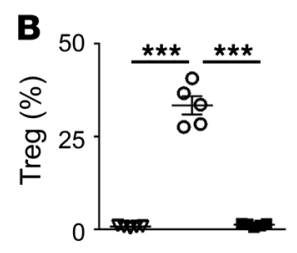

$\nabla$ Medium alone
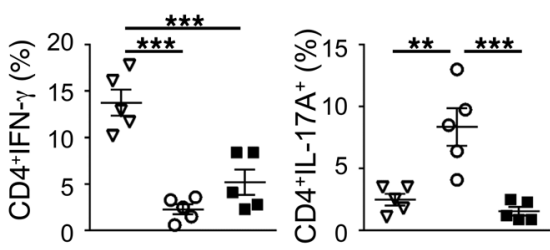

PR3

C
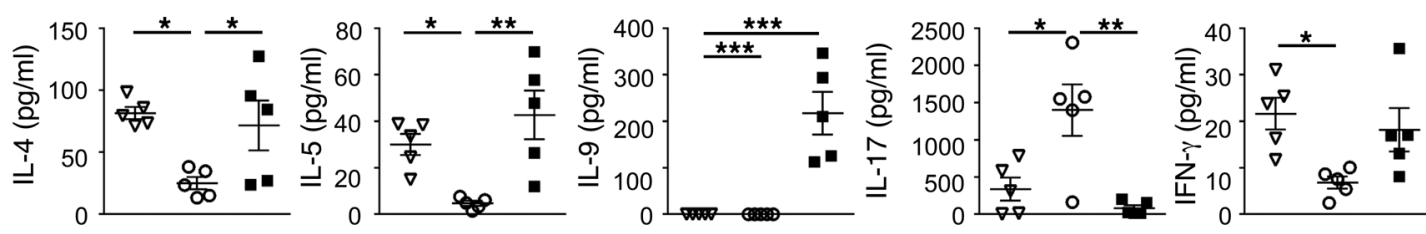

Figure 5. pDCs exposed in vitro to a PR3-induced microenvironment triggered a skewed Th2/Th9 cell distribution. (A) Representative dot plots demonstrating FOXP3 expression in CD4 ${ }^{+}$CD25 $5^{+} \mathrm{T}$ cells and IFN- $\gamma$ and IL-17A expression in CD4 ${ }^{+} \mathrm{T}$ cells. Flow cytometry analysis of naive CD4 ${ }^{+} \mathrm{CD} 25^{-}$OVAspecific OTII T cells cultured for 4 days with pDCs preexposed to macrophage supernatants generated from medium alone (white triangles), apoptotic controls (white circles), or apoptotic PR3-expressing cells (black squares). (B) Percentages of Tregs (CD4+CD25+FOXP3+), Th1 cells (CD4+IFN- $\left.\gamma^{+}\right)$, and Th17 cells (CD4+IL-17A+) obtained following pDCs cocultured with naive T cells. (C) IL-4, IL-5, IL-9, IL-17, and IFN- $\gamma$ secretion measured in media collected from the coculture experiments using a Luminex assay. Values are shown as mean $\pm \mathrm{SEM} ; n=5$ mice per group, each parameter determined in triplicates. ${ }^{*} P<0.05$; ${ }^{* *} P<0.01 ;{ }^{* * *} P<0.001$. Significant differences between groups were determined by multicomparison ANOVA (B and $\left.\mathbf{C}\right)$. 

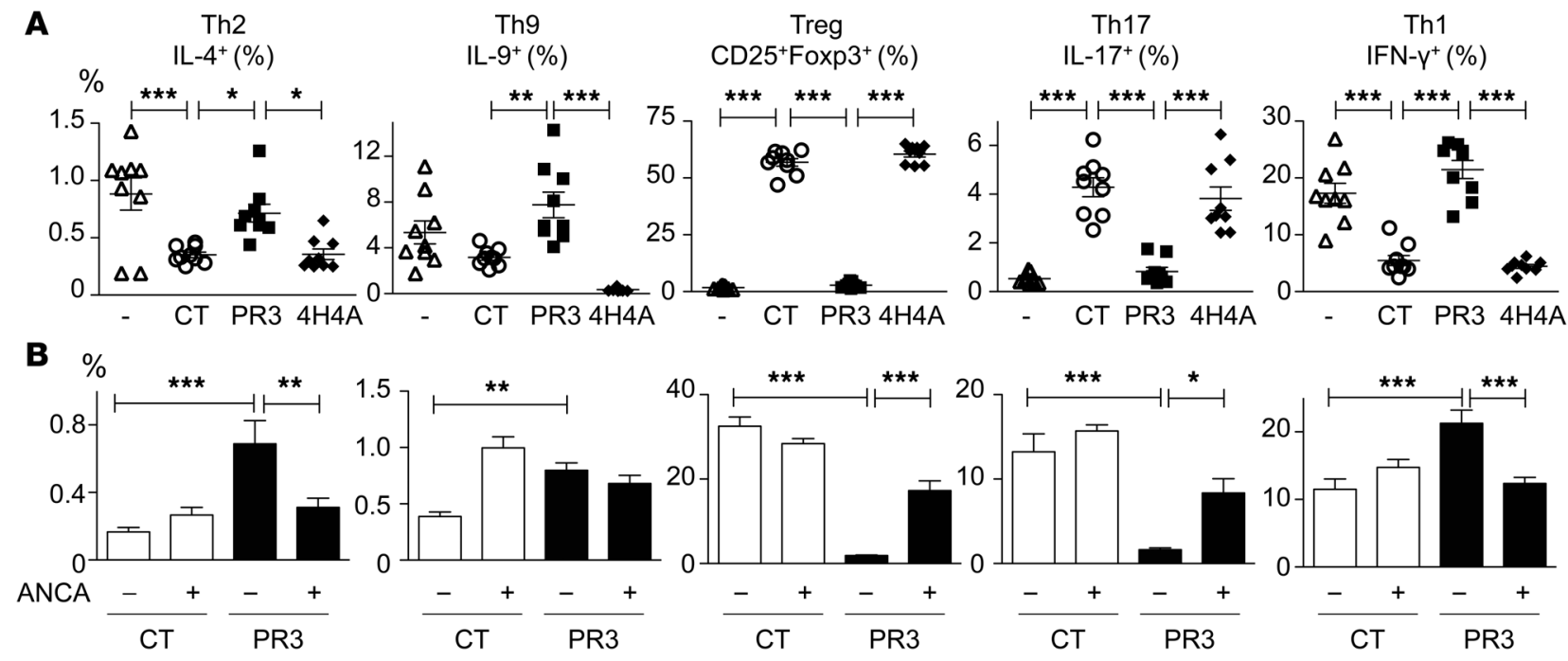

Figure 6. pDCs exposed in vivo to a PR3-induced microenvironment triggered the production of Th9 cells, and addition of anti-PR3 ANCA facilitated Th17 cell polarization. (A) pDCs isolated from mice following the i.v. injection of vehicle (white triangles), apoptotic controls (white circles), PR3- (black squares), or PR3/4H4A-expressing cells (black diamonds) were cultured with naive CD25-CD4+ ${ }^{-}$cells from OTII/Rag $1^{1 /-}$ mice in the presence of OVA peptide. Flow cytometry analysis was performed 4 days later, and the percentages of Tregs (CD4+CD25+FOXP3+), Th9 (CD4+IL-9+), Th2 (CD4+IL-4+), Th1 (CD4+IFN- $\left.\gamma^{+}\right)$, and Th17 (CD4+IL-17A+) cells determined. (B) The above experiments were also performed with the concomitant injection of anti-PR3 ANCA or control IgG in the presence of apoptotic control (white bars) or PR3-expressing cells (black bars). T cell polarization was analyzed using flow cytometry. Data are presented as mean \pm SEM. $n=3$ mice per group, each mice studied in triplicate. Significant differences between groups were determined by ANOVA with multicomparison analysis. ${ }^{*} P<0.05 ;{ }^{* *} P<0.01 ;{ }^{* *} P<0.001$. The experiment presented in $\mathbf{A}$ was reproduced twice with identical results.

(Figure 9E). Pertinently, following activation by either PMA or the bacterial peptide $N$-formyl-methionyl-leucyl-phenylalanine (f-MLF) (2 potent degranulating agents), release of soluble PR3 was increased in GPA patients compared with healthy controls (Figure 9F).

Gene expression analysis was performed on human neutrophils isolated from healthy controls, and PR3 mRNA was found to be constitutively present. Neutrophils from both GPA patients and G-CSF-treated donors displayed PR3 transcripts levels equivalent to those from controls (Supplemental Figure 3), a finding consistent with a recently published paper (42), and other genes known to be increased in both GPA and G-CSF-treated donors, including GADD45A, were increased compared with controls in these experiments (43). Furthermore, treatment of purified neutrophils with G-CSF did not increase PR3 mRNA, despite inducing expression of $\mathrm{B}$ cell activating factor $(B A F F)$, as expected (ref. 44 and Supplemental Figure 3). In addition, G-CSF treatment did not affect PR3 membrane expression on neutrophils from controls or GPA patients (Supplemental Figure 4). Taken together, these observations strongly suggest that increased PR3 protein present in mature circulating neutrophils from GPA patients and G-CSFtreated donors (Figure 9E) may originate from increased $P R 3$ transcription in neutrophil precursors within BM (41). These data also excluded the possibility that upregulation of PR3 protein in GPA patients or G-CSF-treated donors was derived from a direct G-CSF-mediated effect on mature circulating neutrophils.

\section{Discussion}

Apoptotic cells play an essential role in promoting the resolution of inflammation, and their clearance by macrophages has an immunosuppressive effect (32). Here, we propose a paradigm in which anti-PR3-associated necrotizing vasculitis can be consid- ered as an immune deficiency characterized by an impaired antiinflammatory response to apoptotic cell clearance. This is a major advance in the understanding of the pathophysiological mechanisms in ANCA-associated vasculitis, which is typical of a nonresolving inflammation (19), linking the aberrant expression of the autoantigen PR3 to the autoimmune dysregulation typical of GPA. Indeed, granulomatous lesions, which can be located within any tissue (1), are characterized by the presence of numerous neutrophils and macrophages associated with $\mathrm{B}$ cells and $\mathrm{CD} 4^{+} \mathrm{CD} 28^{-}$ $\mathrm{T}$ cells, which are a major source of IFN- $\gamma(45)$.

In this study, we have discovered that membrane PR3 on apoptotic cells activates the IL-1R1/MyD88 signaling pathway, thus acting as a danger signal. Membrane expression of PR3 on apoptotic cells can delay their clearance by macrophages by acting as a "don't-eat-me" signal (14), which in turn favors the accumulation of dead cells at the site of inflammation. Here, we show that membrane PR3 induced by apoptosis is independent of both basal membrane expression and percentage of neutrophil-expressing PR3 (9). Furthermore, proteins able to associate with membrane PR3 during apoptosis differ from those associated with PR3 under basal conditions and this provides an explanation as to why apoptotic neutrophils expressing PR3 are pathogenic and possess immunomodulatory properties (46)

Phagocytosis of apoptotic cells expressing PR3 contributed to the maintenance of inflammation through the secretion of chemokines such as MCP-1, KC, RANTES, MIP-1 $\alpha$, and MIP- $1 \beta$. These chemokines are implicated in the recruitment of neutrophils and monocytes, and high levels of MIP- $1 \alpha$, MIP- $1 \beta$, and RANTES in the lungs of GPA patients have been documented (47, 48). MCP-1 secreted by macrophages may be particularly important, as this protein has been implicated in granuloma formation in a glucan- 
A

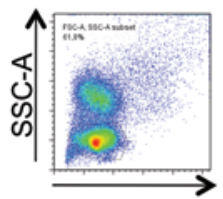

FSC-A

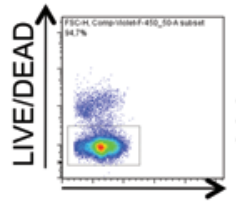

FSC-H

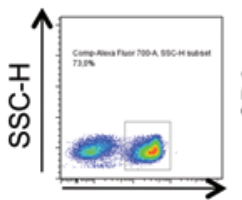

CD3

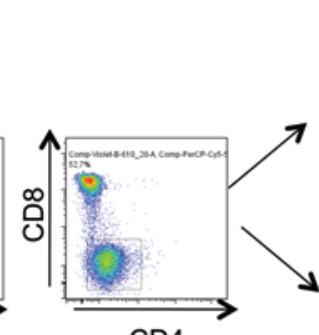

CD4

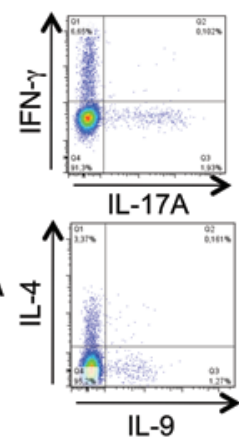

B

$\square \mathrm{HC} \quad \square$ GPA

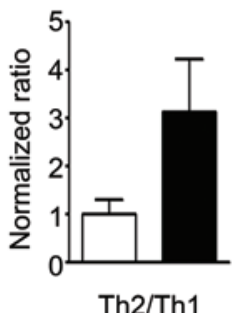

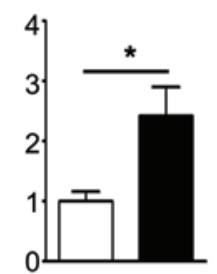

Th17/Th1

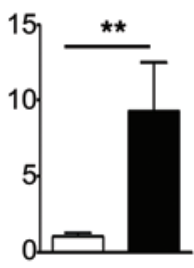

Th9/Th1

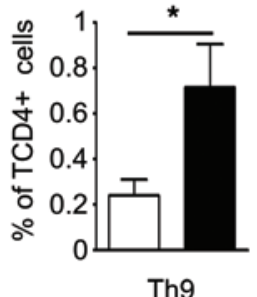

Th9
D

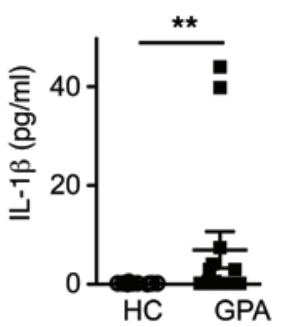

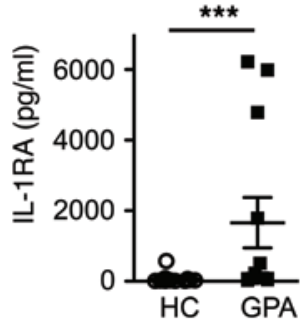
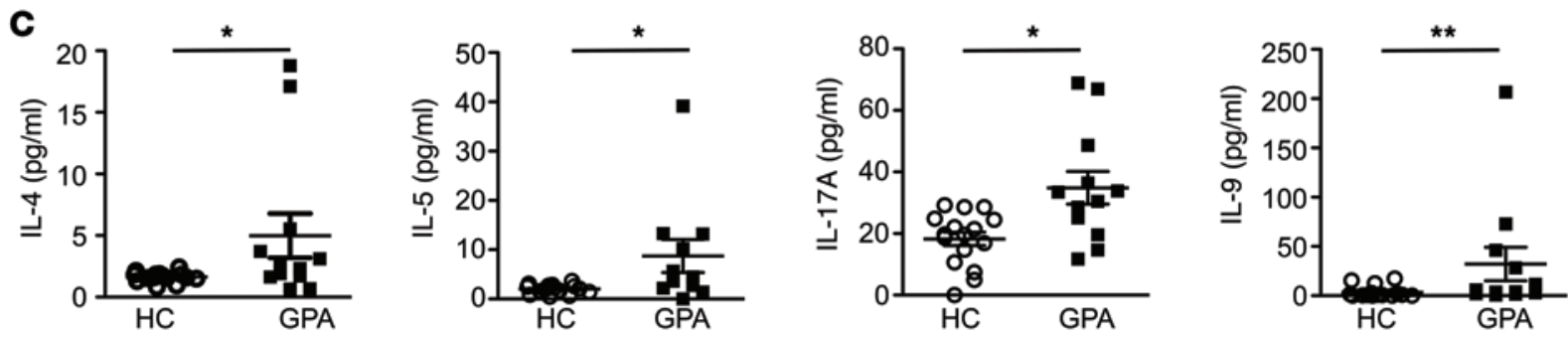

Figure 7. CD4 ${ }^{+}$T cells from GPA patients display a skewed Th9 cell distribution. (A) Representative dot plots demonstrating the gating strategy used to identify Th1, Th2, Th17, and Th9 cells in human PBMCs. (B) The normalized ratio of Th2/Th1, Th17/Th1 and Th9/Th1 in circulating CD4+ T cells was determined in both healthy controls (white bars) and GPA patients (black bars). The percentages of Th9 cells were also calculated ( $n=5$ healthy controls and $n=6$ GPA). (C and D) Sera from healthy controls $(n=16)$ and GPA patients $(n=12)$ were assessed for IL-4, IL-5, IL-17A, IL-9, and for IL-1 $\beta$ and IL-1RA using a Luminex assay. Data are presented as mean $\pm \mathrm{SEM}$. ${ }^{*} P<0.05 ;{ }^{* *} P<0.01 ;{ }^{* *} P<0.001$. Significant differences between groups were determined by multiple comparisons ANOVA (B-D).

induced granulomatous vasculitis (49). The proinflammatory signature was dependent on the presence of PR3 at the membrane of apoptotic cells, as the membrane anchorage-deficient mutant failed to induce a proinflammatory response and resulted in the same antiinflammatory signature observed with control apoptotic cells $(28,34,35,50)$. The enzymatic activity of PR3 was also required to induce a proinflammatory response in macrophages. The phagocytosis of apoptotic cells expressing PR3 strongly induced the expression of iNOS in macrophages, and this represented a shift away from the characteristic proresolving phenotype following efferocytosis $(51,52)$. Furthermore, macrophages from Nos2 ${ }^{--}$mice failed to show the proinflammatory response associated with the phagocytosis of apoptotic cells expressing PR3, demonstrating that NO, a key modulator of macrophage activation (53), was involved in this pathway. Altogether, the proinflammatory chemokine and cytokine production, iNOS activation, and decreased expression of $\mathrm{CD} 206$ in $\mathrm{F} 4 / 80^{+}$macrophages following the phagocytosis of apoptotic cells expressing PR3 supported the hypothesis that membrane-associated and enzymatically active PR3 induced a shift in macrophage polarization toward an M1 profile.
Experiments performed with macrophages obtained from IL-1R1- and MyD88-deficient mice revealed that the proinflammatory effects of PR3 were due, at least in part, to the IL-1R1/ MyD88-dependent signaling pathway. While the proinflammatory effects of PR3 are well documented, for example, through its ability to cleave antiinflammatory proteins such as annexin A1, activate proinflammatory cytokines $(54,55)$, or cleave the protease-activated receptor 2 (PAR2) on DCs (56), to date, no research has made a direct connection between PR3 and immune dysregulation. For the first time, to our knowledge, we establish that IL-1R1 is essential in the proinflammatory response elicited by PR3 expressed on apoptotic cells. Notably, PR3 can process the inactive pro-IL-1 $\beta$ into the bioactive IL-1 $\beta$ in monocytes or macrophages (57). In an experimental model of glomerulonephritis induced with anti-MPO ANCA, generation of IL- $1 \beta$ was critical in pathogenesis, and the receptor antagonist anakinra protected animals against glomerulonephritis (58). While the exact mechanism by which PR3 is recognized remains unknown, our results show that PR3 can affect the IL-1 receptor signaling pathway, presumably but not exclusively through 
A

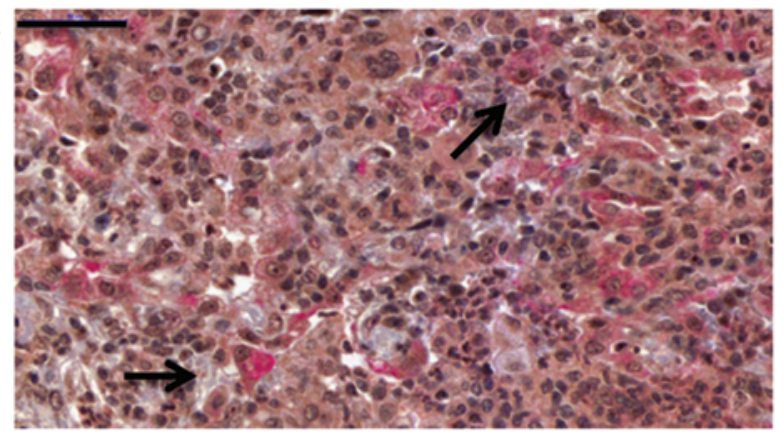

B

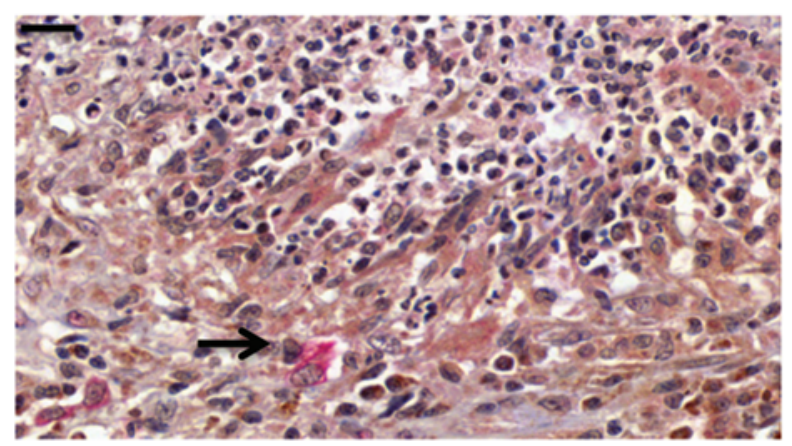

C
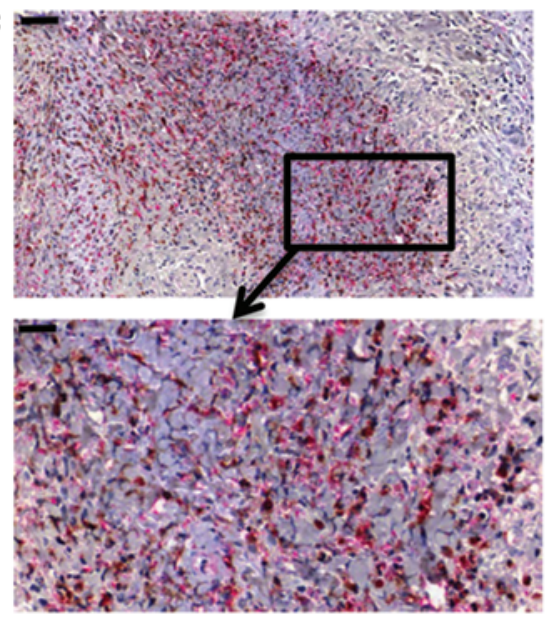

D

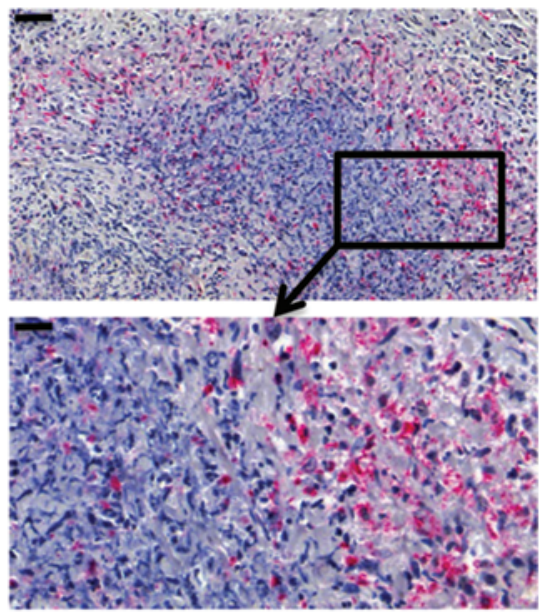

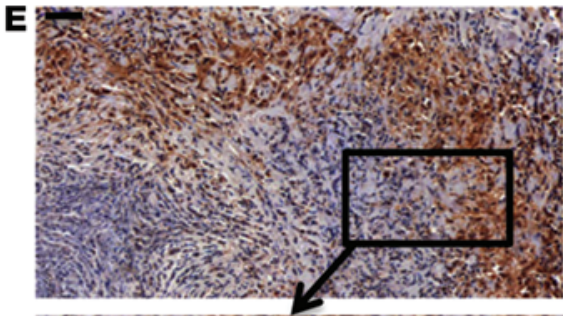

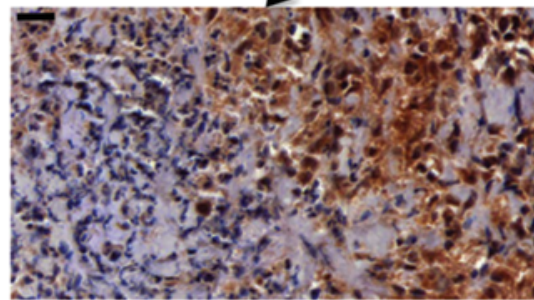

Figure 8. Neutrophils, pDCs, and macrophages were located in granulomatous inflammation from GPA patients. (A) Double-positive CD123 (red staining) and IFN- $\alpha$ cells (brown staining) were located within the granulomatous lesion of lung tissue from GPA patients, and nuclei were counterstained in blue (black arrows). (B) IFN- $\alpha^{+}$(brown staining) and $\mathrm{CD}_{123^{+}}$cells (red staining) were detected at the edge of a neutrophilic abscess in GPA (black arrow). (C-E) Serial sections of a neutrophilic microabscess showed PR3 (C, brown staining) and LL37 cells (C, red staining) surrounded by CD68+ macrophages ( $\mathbf{D}$, red staining) and IFN- $\alpha^{+}$cells (E, brown staining). Lower panels depict higher magnification of the area inside the box in the upper panels. Data display typical images representative of 6 GPA patients. Scale bars: $50 \mu \mathrm{m}$ (A, C-E, upper panels); $20 \mu \mathrm{m}$ (B, C-E, lower panels).

its ability to cleave pro-IL-1 $\beta$ (59). Little is known about the role of members of the IL-1 family in efferocytosis. Release of IL- $1 \alpha$ from endothelium-derived apoptotic bodies has been reported to induce neutrophil recruitment in vasculitis (60). Furthermore, IL-1 signaling is critical for the control of neutrophil apoptosis (61) and for the formation of autoantibodies and generation of pathogenic T cells in lupus (62). Accordingly, we have observed increased levels of IL- $\beta$ and IL-1RA in the sera of GPA patients compared with healthy controls. Therefore, we propose that recognition of membrane PR3 expressed on apoptotic neutrophils in GPA patients facilitates the maintenance of a proinflammatory response via the IL-1R1/MyD88 signaling pathway.

Interestingly, macrophage efferocytosis of apoptotic cells expressing PR3 drove strong secretion of G-CSF. PR3 is a G-CSFresponsive gene involved in myeloid cell differentiation (3), and this may have implications for GPA, as various granulopoiesis genes are dysregulated in neutrophils from these patients (12). A recent report has pointed to the existence of several forms of PR3 transcripts in leukocytes (63). Here, we show that neutrophils from GPA patients overexpressed PR3. Interestingly, these patients have higher concentrations of serum G-CSF compared with healthy controls (64) and increased levels of G-CSF have also been reported in the bronchoalveolar lavage of GPA patients
(65). According to our data, it is possible that the increased expression of PR3 protein in mature circulating neutrophils from GPA patients and G-CSF-treated donors may originate from increased $P R 3$ transcription in neutrophil precursors within BM. This notion is supported by the fact that mature neutrophils treated with G-CSF did not upregulate PR3 transcript levels or membrane PR3 expression.

We demonstrate for what we believe is the first time that pDCs exposed to apoptotic cells expressing membrane PR3 induced the generation of Th2 and Th9 cells, while anti-PR3 ANCA polarized this response toward a Th17 cell distribution. Depending on the context, pDCs are able to direct either Th1 or Th2 polarization (24). Further, TGF- $\beta$-treated pDCs can facilitate Th17 polarization, which is mediated by IL-6 secretion (36), and in a TGF- $\beta$-rich environment, pDCs are associated with IL-4 production and Th9 polarization in pathologies such as systemic sclerosis (66). In ANCA-associated vasculitis, the number of circulating pDCs is decreased during the active phase of the disease, with increased CD62L expression suggesting these cells may be recruited to lymphoid organs where they can interact with other immune cells such as macrophages (67). By coculturing pDCs preexposed to control apoptotic cells in vivo with naive $\mathrm{CD} 4^{+}$ T cells, we observed that the TGF- $\beta$-rich environment generated 
A

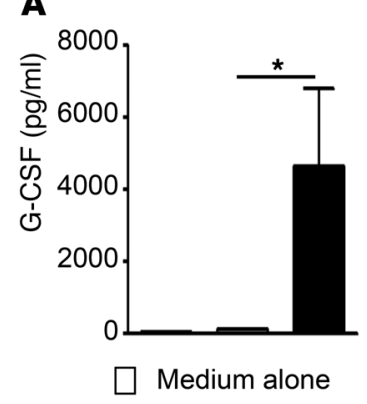

$\mathbf{E}$
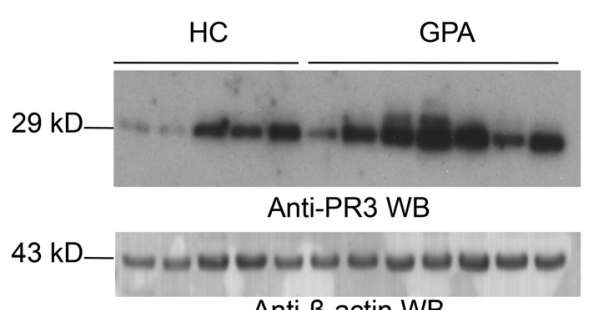

Anti- $\beta$-actin WB

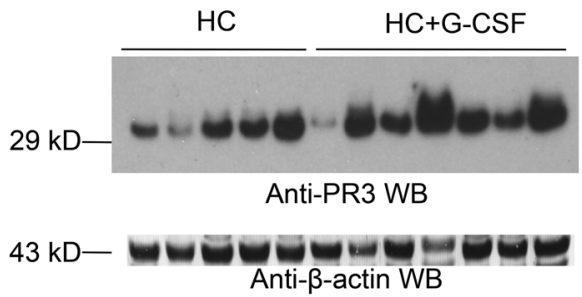

B

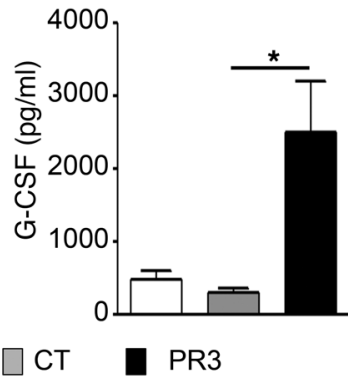

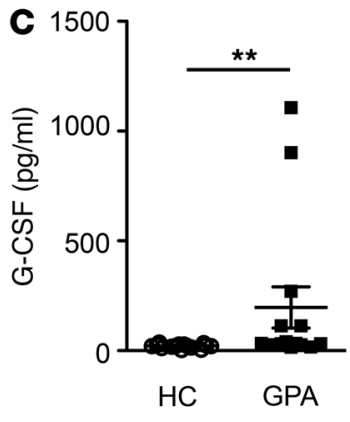

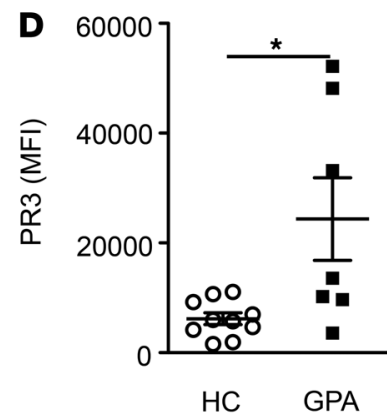

$\mathbf{F}$
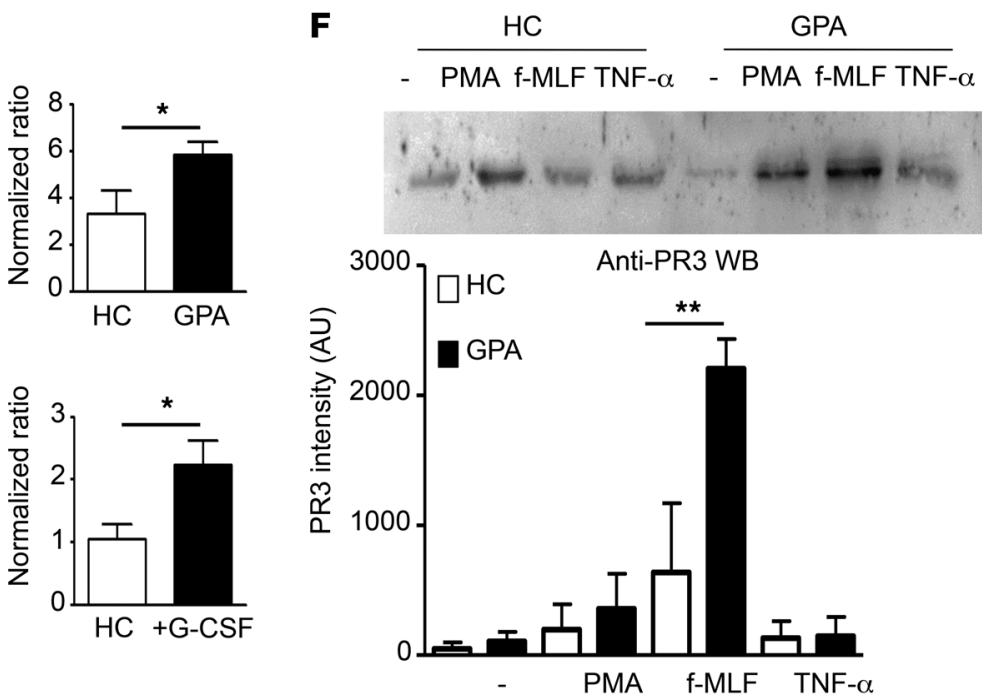

Figure 9. Increased G-CSF secretion by macrophages and T cells was associated with increased expression of PR3 in mature neutrophils. (A) G-CSF concentrations in supernatants from macrophages 24 hours after exposure to medium alone (white bars), apoptotic control cells (gray bars), and PR3-expressing (black bars) cells or (B) G-CSF concentrations in supernatants from pDCs preexposed to the above macrophage supernatants and cocultured with T cells ( $n=4$ mice per group, each concentration was determined in triplicate). (C) G-CSF concentrations in sera from healthy controls ( $n=16)$ and CPA patients $(n=14)$. (D) Intracellular content of PR3 in neutrophils from healthy controls $(n=11)$ or GPA patients $(n=9)$ determined by flow cytometry analysis. (E) Western blot analysis of PR3 expression in neutrophils from healthy controls $(n=5)$ and GPA patients $(n=7)$ (upper panel). Results were quantified by densitometry and normalized to $\beta$-actin loading control. Values represent mean \pm SEM. Similarly, Western blot analysis (lower panel) was also performed on neutrophils from healthy controls $(n=5)$ and healthy controls treated with recombinant G-CSF $(n=7)$. Results were quantified as above. (F) Western blot analysis of PR3 in supernatants of neutrophils treated with PMA, TNF- $\alpha$, or f-MLF. A representative experiment is shown in the upper panel. The quantification of PR3 by densitometry of all the Western blots performed (healthy controls, $n=4$; GPA patients, $n=5$ ) is shown in the lower panel. Values represent mean \pm SEM. Significant differences between groups were determined by ANOVA (A and F) or Mann-Whitney $U$ test $(\mathbf{C}-\mathbf{E}) .{ }^{*} P<0.05 ;{ }^{* *} P<0.01$.

during efferocytosis induced Tregs, an observation in accordance with the literature (36). On the contrary, the microenvironment induced by apoptotic cells expressing PR3 skewed the T helper profile toward a Th2/Th9 and to a lesser extent Th1 profile. Surprisingly, PR3 expressed on apoptotic cells did not affect TGF- $\beta$ secretion. Since it has been reported that PR3 can interact with TGF- $\beta$ (68), this may affect either the detection and/or the biologic activity of TGF- $\beta$. Using our experimental protocol, we demonstrated that addition of anti-PR3 ANCA resulted in Th17 cell production. To our knowledge, this is the first report showing that an autoantigen and its autoantibody can modify Th17 polarization. While PR3 membrane expression on apoptotic neutrophils is not exclusive to GPA, the presence of ANCA targeting PR3 is indeed a feature of this disease.

PBMCs from active, untreated systemic GPA patients also displayed skewed Th9 and Th17 responses. Th9 cells are a subpopulation of $\mathrm{CD}^{+} \mathrm{T}$ cells, which secrete IL- 9 and are implicated in the pathogenesis of allergic diseases such as asthma (69).
Persistent expansion of activated circulating $\mathrm{T}$ cells displaying an effector memory phenotype and abundance of such $\mathrm{T}$ cells in inflammatory lesions suggest a fundamental alteration of $\mathrm{T}$ cell responses in GPA $(45,70,71)$; increased frequencies of circulating Th1 and Th17 cells have also been reported $(45,72)$. Previous studies reported that cytokine responses in peripheral blood $\mathrm{T}$ cells are skewed toward an increase in Th2, Th17, and Th22 cells following conventional in vitro stimulation of PBMCs with PR3 in GPA $(73,74)$. In inflammatory lesions from kidney biopsies, mast cells and $\mathrm{T}$ cells appear to be a source of IL-17 $(45,75)$; however, this observation requires further investigation due to the failure of the antibody used to recognize IL-17 specifically (76). Since Th17 cells and production of their effector cytokines IL-17A, IL-17F, IL-21, and CCL20 may contribute to the pathogenesis of GPA $(77,78)$, targeting these cells could have therapeutic potential. The interactions between pDCs and $\mathrm{T}$ cells not only occur at a systemic level, but also locally within the granulomatous lesions, a hallmark of GPA. Within these 


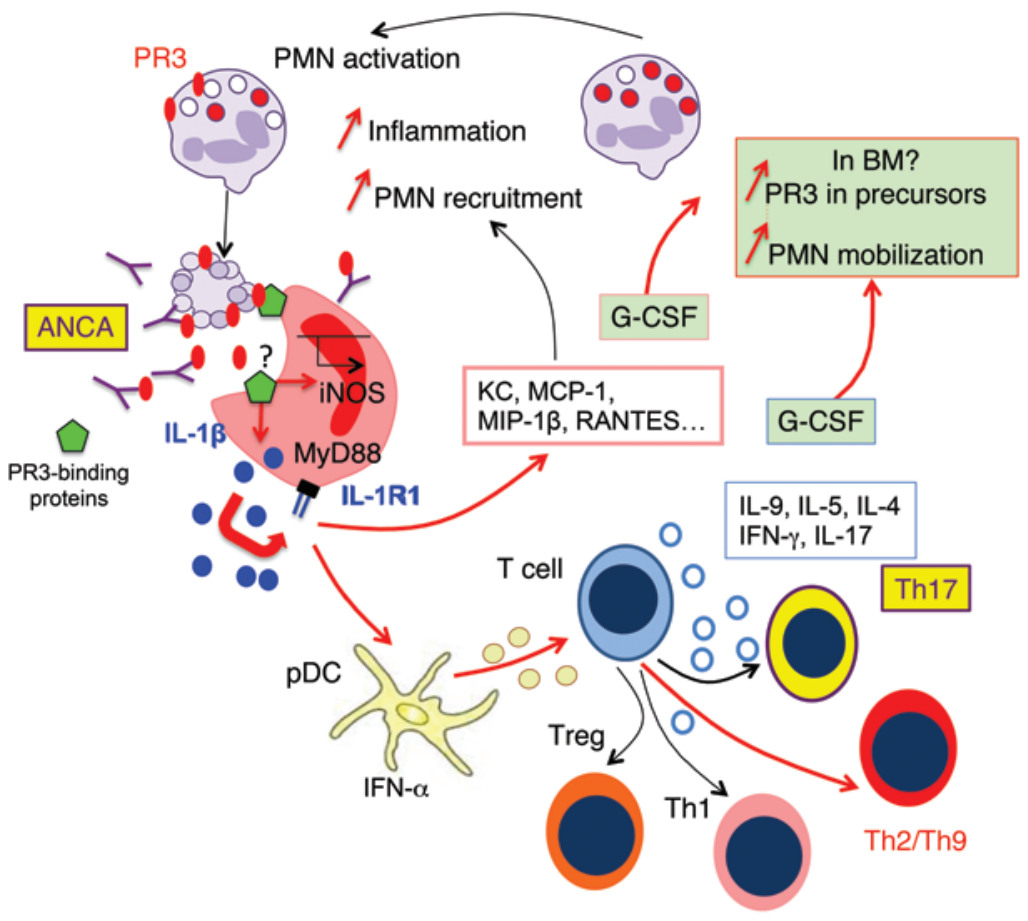

Figure 10. Apoptotic cells expressing phosphatidylserineassociated PR3 induce proinflammatory responses and favor Th9 polarization: in vivo switch to Th17 in the presence of PR3 ANCA. Phagocytosis of apoptotic cells expressing phosphatidylserine-associated PR3 stimulates the production of inflammatory chemokines by macrophages (depicted in red) through the IL-1R1/MyD88 signaling pathway and NO production, thus promoting the recruitment of inflammatory cells expressing PR3, including neutrophils and monocytes. This process acts as an amplification loop and perpetuates inflammation. The presence of phosphatidylserine-associated PR3 on apoptotic cells generates a proinflammatory microenvironment, which facilitates the production of Th2/Th9- and Th1-activated CD4+ $T$ cells through the interaction between pDCs and naive T cells. Importantly, the presence of anti-PR3 ANCA induces Th17 cell production. Increased G-CSF production by macrophages and by $T$ cells may result in an increased PR3 biosynthesis and mobilization of neutrophils from BM, suggesting that PR3 participates in an autoamplification loop, which in turn sustains inflammation. Expression of phosphatidylserineassociated PR3 on apoptotic neutrophils, which prevents the resolution of inflammation, appears to be a key element in the pathogenesis of GPA. PMN, neutrophils. lesions, we observed IFN- $\alpha^{+} \mathrm{CD} 123^{+} \mathrm{pDCs}$ within close proximity to macrophages and PR3 ${ }^{+}$neutrophils as well as $\mathrm{T}$ lymphocytes. Detection of $\mathrm{PR}^{+}$caspase $3^{+}$cells provided further evidence that apoptotic neutrophils are located within this granulomatous inflammation (1) and can interact with the major players driving granuloma formation. Together, our results provide evidence that the phagocytosis of apoptotic cells expressing PR3 can act as a trigger in reprogramming the immune response away from a proresolutive toward a proinflammatory phenotype. This affects not only macrophages, but also the interaction between pDCs and $\mathrm{T}$ cells and is perpetuated by the G-CSF-driven autoamplification of PR3 expression. Addition of anti-PR3 ANCA into this system was able to facilitate the induction of Th17 cells. We propose that PR3, through both its enzymatic activity and membrane expression, acts as a "danger" signal, generating a proinflammatory microenvironment through the IL-1R1/MyD88 and iNOS signaling pathways (Figure 10).

In conclusion, we unambiguously demonstrate that the presence of the autoantigen PR3 on the membrane of apoptotic cells alters the immunosuppressive effect of apoptotic cell efferocytosis and promotes sustained inflammation. Our data strongly support the notion that PR3 subverts immune silencing and orchestrates an autoimmune dysregulation through several mechanisms, including macrophage polarization (79). This insight into the disease must be considered and harnessed to successfully develop new animal models of GPA, which are severely lacking. Finally, in vivo experiments with an inactive PR3 mutant clearly demonstrate that the proinflammatory effect of PR3 involves the cleavage of target protein or proteins. These data exploring the mechanisms of PR3-induced inflammation are essential for developing future novel therapeutic strategies, which could aim to inhibit the serine protease activity of PR3 or target specific immune cells.

\section{Methods}

Human healthy controls and GPA patients. Human neutrophils and PBMCs from healthy donors (Etablissement Français du Sang, SaintAntoine Hospital, Paris France), G-CSF-treated healthy donors (Service des Biothérapies, Necker Hospital) (80), and GPA patients were isolated from EDTA-anticoagulated blood as previously described (21). Patients with GPA fulfilled the Chapel Hill Consensus Conference (CHCC) definition for GPA (81) and were included in the prospective multicenter Neutrovasc study (Programme Hospitalier de Recherche Clinique PHRC no. 2010-AOM10055, primary investigator Luc Mouthon). All patients exhibited anti-PR3 ANCA and had not received any glucocorticoid or immunosuppressive treatment, except 2 who presented with a relapse on maintenance therapy and had active GPA as determined by a Birmingham vasculitis activity score (BVAS 3.0) of 3 or more (ref. 82 and Supplemental Table 1). Sera were collected from healthy donors and GPA patients and stored at $-80^{\circ} \mathrm{C}$ until use. For histopathological analysis, sinunasal and lung biopsies from 6 patients with GPA fulfilling the CHCC definition for GPA (81-84) were analyzed (Supplemental Table 2).

Human blood cell isolation and analysis. Human neutrophil isolation using a Ficoll gradient and measurement of PR3 and CD11b (FITC, Beckman Coulter) membrane expression was performed as previously described $(9,21)$. Briefly, detection of PR3 on human neutrophils in the absence or in the presence of TNF- $\alpha(10 \mathrm{ng} / \mathrm{ml})$ was performed using a mouse anti-PR3 (clone CLB12.8; Sanquin), followed by incubation with a fluorochrome-conjugated anti-mouse IgG (Immunotech). For induction of apoptosis, isolated neutrophils were incubated for 16 hours at $37^{\circ} \mathrm{C}$ and cells were labeled with annexin $\mathrm{V}$ and 7-AAD to assess apoptotic versus necrotic cells, respectively, as previously described (21). Labeled cells were acquired and analyzed on a BD Accuri flow cytometer (BD Biosciences).

For degranulation experiments, neutrophils isolated from healthy donors or GPA patients were resuspended at a concentration of $2 \times 10^{7}$ 
cells/ml in PBS in the presence of PMA (100 ng/ml), f-MLF (10 nM), or TNF- $\alpha(50 \mathrm{ng} / \mathrm{ml})$ for 15 minutes at $37^{\circ} \mathrm{C}$. Following centrifugation $(10 \mathrm{~min}$ at $2000 \mathrm{~g}$ ), $40 \mu \mathrm{l}$ of supernatants corresponding to the degranulation of 80,000 neutrophils was analyzed by Western blot for the presence of PR3, as previously described $(8,9)$.

Human T cells from PBMCs were labeled using monoclonal antibodies anti-CD3 (AF 700 clone UCHT1; BD Biosciences), anti-CD4 (Brilliant Violet 605 clone RPA-T4; BD Biosciences), and anti-CD8 (PerCP-Cy5.5 clone RPA-T8; BD Biosciences). Cells were next permeabilized and incubated with monoclonal antibodies against cytokines IL-4 (APC clone 8D4-8; eBioscience), IL-9 (PE clone MH9D1; eBioscience), IL-17A (PE clone N49-653; BD Biosciences), and IFN- $\gamma$ (FITC clone B27; BD Biosciences) and the corresponding isotypic controls, IgG1 (FITC clone MOPC-21; BD Biosciences), IgG1 (APC clone P3.6.2.8.1; eBioscience), and IgG2a (PE clone eBM2a; eBioscience). Labeled cells were acquired on a FACSCanto II flow cytometer using FACSDiva software (BD Biosciences). Flow cytometry data acquisition and data analysis were performed at the Cochin Cytometry and Immunobiology Facility (Cochin Institute).

Immunohistochemistry and immunofluorescence analysis. Single or double immunohistochemistry of anonymized formalin-fixed paraffin-embedded sinunasal or lung tissue sections from active GPA patients $(n=6)$ was performed as previously described (37), using primary antibodies against CD123 (polyclonal rabbit anti-human, Thermo Fisher Scientific), IFN- $\alpha$ (mouse anti-human, MMHA-11; R\&D Systems), LL37 (rabbit anti-human; Acris Antibodies), and CD68 (mouse anti-human, PGM1; Dako). Double immunofluorescence staining was also performed (37) using primary antibodies against PR3 (mouse anti-human, WGM2; gift of E. Csernok (Klinikum Bad Bramstedt, Bad Bramstedt, Germany) and cleaved caspase 3 (rabbit anti-human; Cell Signaling) (36).

In vivo phagocytosis of apoptotic $R B L$ cells by murine macrophages. RBL cells were cultured as previously described (30). Recombinant human PR3, the membrane anchorage-deficient mutant (PR3/4H4A), or the enzymatic inactive mutant (PR3/S203A) was expressed in stably transfected RBL cells as previously described (31). Apoptosis of RBL cells was induced by either UV-B (5 min, $\mathrm{l}=312 \mathrm{~nm}, 0.68 \mathrm{~mW} / \mathrm{cm}^{2}$ for 16 hours $)$ or gliotoxin $(2 \mu \mathrm{g} / \mathrm{ml}$ for 24 hours) (14) as indicated and evaluated using flow cytometry with annexin V-PE/7-AAD (BD Biosciences).

Phagocytosis by resident macrophages was induced in C57BL/6J mice by i.p. injection of $1 \times 10^{7}$ apoptotic RBL cells (controls, PR3, or S203APR3 mutant). Peritoneal fluid was collected after 2 hours and frozen at $-20^{\circ} \mathrm{C}$ before measuring proinflammatory cytokine and chemokine secretion using a proinflammatory mouse cytokine assay (MSD). MCP-1 and G-CSF were measured using Duoset (R\&D Systems). Alternatively, cells were harvested from the peritoneal cavity 24 hours after apoptotic cell injection and surface markers on macrophages $\mathrm{F} 4 / 80^{\text {hi }}$ (APC or PE, clone BM8; eBioscience) analyzed by flow cytometry using the following monoclonal antibodies: CD11b (APC clone M1/70; eBioscience), CD11c (FITC, clone HL3; BD Biosciences), I-A/I-E (APC, clone MS/114.15.3; eBioscience), CD206 (APC, R\&D Systems), CD23 (PE-Cy7 clone 83B4, BD Biosciences), CD16/CD32 (V450 clone 2.4G2, BD Biosciences), and TLR4 (PE-Cy, clone MTS510, eBioscience). Labeled cells were acquired on a FACSCanto II flow cytometer using FACSDiva software (BD Biosciences).
Phagocytosis by inflammatory macrophages was evaluated by injecting $1 \times 10^{7}$ apoptotic RBL cells (controls or PR3) or vehicle (PBS) 72 hours after the initial injection of thioglycolate. Cytokines (IL-1 $\beta$, IL-4, IL-5, IL-6, IL-9, IL-12p70, IL-17, IFN- $\gamma$, TNF- $\alpha$ ), growth factors (G-CSF, GM-CSF), and chemokines (MIP-1 $\alpha$, MIP-1 $\beta$, RANTES, MCP-1, $\mathrm{KC}$ ) were measured using a BioPlexPro. Data acquisition and analysis were performed at the Cochin Cytometry and Immunobiology Facility. Macrophage iNOS expression was analyzed by Western blot as previously described (52). Briefly, proteins $(5 \mu \mathrm{g} / \mathrm{lane})$ were separated on $10 \%$ SDS-PAGE and transferred to PVDF membranes (Bio-Tad). Anti-iNOS (ab3523, 1:500; Abcam) was used as the primary antibody followed by an HRP-conjugated secondary antibody (Jackson ImmunoResearch). Blots were washed as above and developed using a WesternBright ECL (Advansta) chemiluminescence kit. Detection of $\beta$-actin was used as a loading control on the same membrane.

In vitro phagocytosis of apoptotic RBL cells by murine macrophages. Macrophages from C57BL/6J female mice between 12 and 20 weeks of age (Charles River Laboratories) were collected 72 hours after i.p. injection of thioglycolate (3\%; BBL Thioglycolate Medium, Brewer Modified; BD) and plated in DMEM supplemented with 10\% heatinactivated FCS, $1 \%$ penicillin/streptomycin, $10 \mathrm{mM}$ HEPES buffer, $10 \mathrm{mM}$ nonessential amino acids, $4 \mathrm{mM}$ L-glutamine, $1 \mathrm{mM}$ sodium pyruvate, and $0.05 \mathrm{mM} 2-\mathrm{ME}$ for 6 hours. Plates were washed multiple times to remove nonadherent cells and exclude eosinophils; purity was assessed with F4/80, CD11b, and CD11c antibodies and found to be greater than $90 \%$. Apoptotic cells (typically annexin V $>80 \%, 7-\mathrm{AAD}<15 \%$ ) were added to macrophage cultures (ratio of 5:1) and phagocytosis allowed to occur for 24 hours. Assessment of phagocytosis was performed by flow cytometry using apoptotic RBL cells stained with TAMRA (Sigma-Aldrich) as previously described (21) to validate that, at the time point used, there were no differences in the phagocytosis rate between the different apoptotic cell lines used (data not shown). The culture medium, termed phagocytosis supernatant, was collected and frozen at $-80^{\circ} \mathrm{C}$ until required. To test the response to apoptotic cells in different strains of mice, including Nos2 $2^{---}$(provided by Armelle Blondel), Tlr2 $2^{-/}, \mathrm{Tlr}^{-/-}$, and $\mathrm{Myd} 88^{-/-}$ (provided by Frédéric Péné (Cochin Institute), and $I l 1 r 1^{-/-}$(provided by Bernhard Ryffel; CNRS UMR 7355, Orléans, France), apoptosis was induced with gliotoxin $(2 \mu \mathrm{g} / \mathrm{ml})$. Macrophages were then stimulated in RPMI with LPS (10 ng/ml, O55B5; Sigma-Aldrich) for a further 24 hours, and culture medium, termed stimulation supernatant, was collected and frozen at $-80^{\circ} \mathrm{C}$.

Murine pDC isolation and coculture with $T$ cells. pDCs were enriched from C57BL/6 BM using an anti-mPDCA1 microbead kit according to the manufacturer's instructions (Miltenyi Biotec). Purity was determined by flow cytometry using anti-Siglec-H (PE; clone eBio440c; eBioscience) and anti-PDCA (allophycocyanin, clone JF05-1C2.4.1; Miltenyi Biotec) in combination with anti-NK1.1 (FITC, clone PK136; eBioscience) and anti-CD19 (APC-Cy7; clone 1D3, eBioscience) and found to be greater than $85 \%$. For each experiment, 10 to 12 mice were used to collect sufficient pDCs. Enriched pDCs were rested overnight in complete medium (RPMI 1640 medium supplemented with 10\% FBS, 1\% penicillin/ streptomycin, $10 \mathrm{mM}$ HEPES, $10 \mathrm{mM}$ nonessential amino acids, $1 \mathrm{mM}$ sodium pyruvate, $4 \mathrm{mM}$ L-glutamine, and $0.05 \mathrm{mM} 2-\mathrm{ME}$ ) in the presence of mouse rIL-3 (10 ng/ml; PeproTech). The cells were exposed to supernatants of macrophages in X-VIVO (Lonza) media for a further 24 hours and extensively washed in PBS. C57BL/6 OTII $\alpha \beta$ TCR-transgenic 
Rag-1-deficient (OTII/Rag1-/-) mice (Center of Immunology of MarseilleLuminy, Marseille, France) expressing only $\mathrm{T}$ cells with OVA-specific $\mathrm{TCR}$, were used to study $\mathrm{CD} 4^{+} \mathrm{T}$ cell polarization (36). pDCs were then cocultured for 4 days with naive $\mathrm{CD} 4^{+} \mathrm{CD} 25^{-} \mathrm{T}$ cells from OTII/Rag1 ${ }^{-1}$ mice in the presence of $\mathrm{OVA}_{323-339}$ peptide $(2 \mathrm{mg} / \mathrm{ml}$; NeoMPS) at a ratio of 1:2. CD $4^{+} \mathrm{CD} 25^{-} \mathrm{T}$ cells were isolated from the thymus of $\mathrm{C} 57 \mathrm{BL} / 6$ mice using the $\mathrm{CD}^{+} \mathrm{CD} 25^{-}$Regulatory $\mathrm{T}$ Cell Isolation Kit (Miltenyi Biotec) ( $>95 \%$ purity). Polarization of $\mathrm{CD}^{+} \mathrm{T}$ cells was determined by flow cytometry analysis with a FACSCanto II using CD3 (FITC, clone 17A2; eBioscience), CD4 (Pacific Blue, clone RM4-5; eBioscience), CD25 (Alexa Fluor 488 or PE, clone PC61.5; eBioscience), IFN- $\gamma$ (PE; clone XMG1.2; BioLegend), IL-17A (allophycocyanin, clone TC 11-18H10.1; BioLegend), and FOXP3 (PE-Cy5, clone FJK-16s; eBioscience) antibodies according to the manufacturer's instructions. For IFN- $\gamma$ and IL-17A intracellular staining, cells were stimulated with PMA $(25 \mathrm{ng} / \mathrm{ml})$ and ionomycin $(1 \mu \mathrm{g} / \mathrm{ml})$ in the presence of brefeldin A for 4 hours.

In vivo sensitization of pDCs by apoptotic cells before isolation. For the in vivo model, mice were injected i.v. with apoptotic cells and spleens collected 48 hours later. pDCs exposed to an apoptotic cell-induced microenvironment in vivo were magnetically sorted from spleens and cocultured with naive $\mathrm{CD} 25^{+} \mathrm{CD} 4^{+} \mathrm{T}$ cells from OTII/Rag1 $1^{-/}$transgenic mice in the presence of $\mathrm{OVA}_{323-339}$ peptide for 4 days. Where required, concomitant i.v. injection of both apoptotic cells and mouse anti-PR3 ANCA (2 mg/mouse, clone CLB12.8, Sanquin) or IgG1 control ( $2 \mathrm{mg} / \mathrm{mouse}$ ) was performed before collecting pDCs and polarization of $\mathrm{CD} 4^{+} \mathrm{T}$ cell was examined as described above.

Statistics. Data are shown as mean \pm SEM. Statistical analyses were performed using Prism GraphPad 5.01 software. Correlations were assessed using Pearson's tests. Comparisons were made using the nonparametric Mann-Whitney $U$ test or multicomparison ANOVA with the Tukey's post test, where appropriate. Differences were considered significant at $P<0.05$.

Study approval. Blood donors gave written informed consent to participate in this study, which was approved by the INSERM Institutional Review Board (NEUTROVASC DR-2012-002) and the Cochin Hospital Ethics Committee. Written consent to participate in the Neutrovasc study was obtained according to the Declaration of Helsinki (CPP authorization no. 2011-12767). For immunohistochemistry analysis of biopsies, written consent from GPA patients was obtained according to the Declaration of Helsinki and study design was approved by the ethics committee of the University of Lübeck (no. 07-058). Animal studies were performed in accordance with European Community Guidelines (VWS authorization no. B-751394). All protocols were approved by the Université Paris Descartes Ethics Committee for Animal Research (CEEA34.VWS.074.12).

\section{Acknowledgments}

The authors acknowledge Muriel Andrieu and Karine Labroquère of the Cochin Cytometry and Immunobiology Facility; Agnès Lebon and the staff of the Animal Care Facilities at the Cochin Institute; investigators of the Neutrovasc study and Séverine Ait el Ghaz-Poignant and Pauline Rivoire for monitoring the data; the excellent technical assistance of Christopher Connor and Céline Candalh for some in vivo experiments and Delphine Ohayon and Judith Everts-Graber for neutrophil experiments; and the Etablissement Français du Sang. This work was supported by individual funding, as follows: the Investissements d'Avenir programme ANR-11-IDEX-0005-02, Sorbonne Paris Cite, LabEx INFLAMEX, Labex LipSTIC (ANR-11-LABX-0021), the Chancellerie des Universités de Paris (Legs Poix VWS), the Programme Hospitalier de Recherche Clinique (Ministry of Health; PHRC no. 2010-AOM10055) and Assistance Publique-Hôpitaux de Paris (AP-HP), the DHU AUTHORS (AP-HP and Paris Descartes University), the FHU INCREASE (Besançon, France), the Région de Franche-Comté and the German Research Foundation CRU170 (to P. Lamprecht and A. Mueller), RTG1727 (to A. Mueller, P. Lamprecht), and EXC306 (to P. Lamprecht). A. Millet was supported by INSERM (poste d'accueil), and K.R. Martin received an Early Career Fellowship from NHMRC (1092602).

Address correspondence to: Véronique Witko-Sarsat, INSERM U1016, Institut Cochin, 27 rue du Faubourg St-Jacques, 75014 Paris, France. Phone: 33.1.40.51.66.56; E-mail: veronique. witko@inserm.fr.
1. Jennette JC, Falk RJ, Hu P, Xiao H. Pathogenesis of antineutrophil cytoplasmic autoantibodyassociated small-vessel vasculitis. Annu Rev Pathol. 2013;8:139-160.

2. Lyons PA, et al. Genetically distinct subsets within ANCA-associated vasculitis. $N$ Engl J Med. 2012;367(3):214-223.

3. Bories D, Raynal MC, Solomon DH, Darzynkiewicz Z, Cayre YE. Down-regulation of a serine protease, myeloblastin, causes growth arrest and differentiation of promyelocytic leukemia cells. Cell. 1989;59(6):959-968.

4. Campanelli D, et al. Cloning of cDNA for proteinase 3: a serine protease, antibiotic, and autoantigen from human neutrophils. JExp Med. 1990;172(6):1709-1715.

5. Dublet B, et al. Cleavage of p21/WAF1/CIP1 by proteinase 3 modulates differentiation of a monocytic cell line. Molecular analysis of the cleavage site. J Biol Chem. 2005;280(34):30242-30253.

6. Just J, et al. Proteinase 3 mRNA expression is induced in monocytes but not in neutrophils of patients with cystic fibrosis. FEBS Lett. 1999;457(3):437-440.

7. Hajjar E, Broemstrup T, Kantari C, Witko-Sarsat V, Reuter N. Structures of human proteinase 3 and neutrophil elastase - so similar yet so different. FEBS J. 2010;277(10):2238-2254.

8. Witko-Sarsat V, et al. Presence of proteinase 3 in secretory vesicles: evidence of a novel, highly mobilizable intracellular pool distinct from azurophil granules. Blood. 1999;94(7):2487-2496.

9. Witko-Sarsat V, et al. A large subset of neutrophils expressing membrane proteinase 3 is a risk factor for vasculitis and rheumatoid arthritis. JAm Soc Nephrol. 1999;10(6):1224-1233.

10. Falk RJ, Terrell RS, Charles LA, Jennette JC. Anti-neutrophil cytoplasmic autoantibodies induce neutrophils to degranulate and produce oxygen radicals in vitro. Proc Natl Acad Sci U S A. 1990;87(11):4115-4119.

11. Kettritz R, Jennette JC, Falk RJ. Crosslinking of ANCA-antigens stimulates superoxide release by human neutrophils. J Am Soc Nephrol.
1997;8(3):386-394.

12. Yang JJ, et al. Circumvention of normal constraints on granule protein gene expression in peripheral blood neutrophils and monocytes of patients with antineutrophil cytoplasmic autoantibody-associated glomerulonephritis. J Am Soc Nephrol. 2004;15(8):2103-2114.

13. Ciavatta DJ, et al. Epigenetic basis for aberrant upregulation of autoantigen genes in humans with ANCA vasculitis. JClin Invest. 2010;120(9):3209-3219.

14. Kantari C, et al. Proteinase 3, the Wegener autoantigen, is externalized during neutrophil apoptosis: evidence for a functional association with phospholipid scramblase 1 and interference with macrophage phagocytosis. Blood. 2007;110(12):4086-4095.

15. Durant S, et al. Apoptosis-induced proteinase 3 membrane expression is independent from degranulation. J Leukoc Biol. 2004;75(1):87-98.

16. Savill JS, Wyllie AH, Henson JE, Walport MJ, Henson PM, Haslett C. Macrophage phago- 
cytosis of aging neutrophils in inflammation. Programmed cell death in the neutrophil leads to its recognition by macrophages. J Clin Invest. 1989;83(3):865-875.

17. Filep JG. Resolution of inflammation: leukocytes and molecular pathways as potential therapeutic targets. Front Immunol. 2013;4:256.

18. Poon IK, Lucas CD, Rossi AG, Ravichandran KS. Apoptotic cell clearance: basic biology and therapeutic potential. Nat Rev Immunol. 2014;14(3):166-180.

19. Nathan C, Ding A. Nonresolving inflammation. Cell. 2010;140(6):871-882.

20. Serhan CN, et al. Resolution of inflammation: state of the art, definitions and terms. FASEB J. 2007;21(2):325-332.

21. Gabillet J, et al. Proteinase 3, the autoantigen in granulomatosis with polyangiitis, associates with calreticulin on apoptotic neutrophils, impairs macrophage phagocytosis, and promotes inflammation. J Immunol. 2012;189(5):2574-2583.

22. Gardai SJ, et al. Cell-surface calreticulin initiates clearance of viable or apoptotic cells through trans-activation of LRP on the phagocyte. Cell. 2005;123(2):321-334.

23. Li J, Park J, Foss D, Goldschneider I. Thymushoming peripheral dendritic cells constitute two of the three major subsets of dendritic cells in the steady-state thymus. J Exp Med. 2009;206(3):607-622.

24. Guery L, Hugues S. Tolerogenic and activatory plasmacytoid dendritic cells in autoimmunity. Front Immunol. 2013;4:59.

25. Capraru D, et al. Expansion of circulating NKG2D+effector memory T-cells and expression of NKG2D-ligand MIC in granulomaous lesions in Wegener's granulomatosis. Clin Immunol. 2008;127(2):144-150.

26. Wilde B, et al. Dendritic cells in renal biopsies of patients with ANCA-associated vasculitis. Nephrol Dial Transplant. 2009;24(7):2151-2156.

27. Martin-Gayo E, Sierra-Filardi E, Corbi AL, Toribio ML. Plasmacytoid dendritic cells resident in human thymus drive natural Treg cell development. Blood. 2010;115(26):5366-5375.

28. Bonnefoy F, et al. Plasmacytoid dendritic cells play a major role in apoptotic leukocyteinduced immune modulation. JImmunol. 2011;186(10):5696-5705.

29. Abdgawad M, et al. Decreased neutrophil apoptosis in quiescent ANCA-associated systemic vasculitis. PLoS One. 2012;7(3):e32439.

30. Witko-Sarsat $\mathrm{V}$, et al. Cleavage of $\mathrm{p} 21$ waf1 by proteinase-3, a myeloid-specific serine protease, potentiates cell proliferation. J Biol Chem. 2002;277(49):47338-47347.

31. Kantari C, et al. Molecular analysis of the membrane insertion domain of proteinase 3 , the Wegener's autoantigen, in RBL cells: implication for its pathogenic activity. J Leukoc Biol. 2011;90(5):941-950.

32. Fadok VA, Bratton DL, Konowal A, Freed PW, Westcott JY, Henson PM. Macrophages that have ingested apoptotic cells in vitro inhibit proinflammatory cytokine production through autocrine/ paracrine mechanisms involving TGF- $\beta$, PGE2, and PAF. JClin Invest. 1998;101(4):890-898.

33. Ariel A, Serhan CN. New lives given by cell death: macrophage differentiation following their encounter with apoptotic leukocytes during the resolution of inflammation. Front Immunol. 2012;3:4.

34. Miyake $\mathrm{Y}$, Asano K, Kaise H, Uemura M, Nakayama M, Tanaka M. Critical role of macrophages in the marginal zone in the suppression of immune responses to apoptotic cell-associated antigens. JClin Invest. 2007;117(8):2268-2278.

35. Kleinclauss F, et al. Intravenous apoptotic spleen cell infusion induces a TGF- $\beta$-dependent regulatory T-cell expansion. Cell Death Differ. 2006;13(1):41-52.

36. Bonnefoy F, et al. TGF- $\beta$-exposed plasmacytoid dendritic cells participate in Th17 commitment. JImmunol. 2011;186(11):6157-6164.

37. Mueller A, et al. Plasma cells within granulomatous inflammation display signs pointing towards autoreactivity and destruction in granulomatosis with polyangiitis. Arthritis Res Ther. 2014;16(1):R55.

38. Grouard G, Rissoan MC, Filgueira L, Durand I, Banchereau J, Liu YJ. The enigmatic plasmacytoid $\mathrm{T}$ cells develop into dendritic cells with interleukin (IL)-3 and CD40-ligand. J Exp Med. 1997;185(6):1101-1111.

39. Rollins-Raval MA, Marafioti T, Swerdlow SH, Roth CG. The number and growth pattern of plasmacytoid dendritic cells vary in different types of reactive lymph nodes: an immunohistochemical study. Hum Pathol. 2013;44(6):1003-1010.

40. Semerad CL, Liu F, Gregory AD, Stumpf K, Link DC. G-CSF is an essential regulator of neutrophil trafficking from the bone marrow to the blood. Immunity. 2002;17(4):413-423.

41. Lutz PG, Moog-Lutz C, Coumau-Gatbois E, Kobari L, Di Gioia Y, Cayre YE. Myeloblastin is a granulocyte colony-stimulating factor-responsive gene conferring factor-independent growth to hematopoietic cells. Proc Natl Acad Sci U S A. 2000;97(4):1601-1606.

42. Hu N, et al. Differential expression of granulopoiesis related genes in neutrophil subsets distinguished by membrane expression of CD177. PLoS One. 2014;9(6):e99671.

43. Alcorta DA, et al. Leukocyte gene expression signatures in antineutrophil cytoplasmic autoantibody and lupus glomerulonephritis. Kidney Int . 2007;72(7):853-864.

44. Scapini P, et al. G-CSF-stimulated neutrophils are a prominent source of functional BLyS. J Exp Med. 2003;197(3):297-302.

45. Komocsi A, et al. Peripheral blood and granuloma CD4(+)CD28(-) T cells are a major source of interferon-gamma and tumor necrosis factor- $\alpha$ in Wegener's granulomatosis. Am J Pathol. 2002;160(5):1717-1724.

46. Witko-Sarsat V, Reuter N, Mouthon L. Interaction of proteinase 3 with its associated partners: implications in the pathogenesis of Wegener's granulomatosis. Curr Opin Rheumatol. 2010;22(1):1-7.

47. Zhou Y, Huang D, Farver C, Hoffman GS. Relative importance of CCR5 and antineutrophil cytoplasmic antibodies in patients with Wegener's granulomatosis. JRheumatol. 2003;30(7):1541-1547.

48. Coulomb-L'Hermine A, et al. Expression of the chemokine RANTES in pulmonary Wegener's granulomatosis. Hum Pathol. 2001;32(3):320-326.

49. Jones ML, Warren JS. Monocyte chemoattractant protein 1 in a rat model of pulmonary granulomatosis. Lab Invest. 1992;66(4):498-503.

50. Poon IK, Lucas CD, Rossi AG, Ravichandran KS. Apoptotic cell clearance: basic biology and therapeutic potential. Nat Rev Immunol. 2014;14(3):166-180.

51. Freire-de-Lima CG, Xiao YQ, Gardai SJ, Bratton DL, Schiemann WP, Henson PM. Apoptotic cells, through transforming growth factor-beta, coordinately induce anti-inflammatory and suppress pro-inflammatory eicosanoid and NO synthesis in murine macrophages. J Biol Chem. 2006;281(50):38376-38384.

52. Schif-Zuck S, Gross N, Assi S, Rostoker R, Serhan $\mathrm{CN}$, Ariel A. Saturated-efferocytosis generates pro-resolving CD11b low macrophages: modulation by resolvins and glucocorticoids. Eur $J$ Immunol. 2011;41(2):366-379.

53. Nathan C, Cunningham-Bussel A. Beyond oxidative stress: an immunologist's guide to reactive oxygen species. Nat Rev Immunol. 2013;13(5):349-361.

54. Vong L, et al. Annexin 1 cleavage in activated neutrophils: a pivotal role for proteinase 3. J Biol Chem. 2007;282(41):29998-30004.

55. Coeshott C, et al. Converting enzyme-independent release of tumor necrosis factor alpha and IL-1 $\beta$ from a stimulated human monocytic cell line in the presence of activated neutrophils or purified proteinase 3. Proc Natl Acad Sci U S A. 1999;96(11):6261-6266.

56. Csernok E, et al. Wegener autoantigen induces maturation of dendritic cells and licenses them for Th1 priming via the protease-activated receptor-2 pathway. Blood. 2006;107(11):4440-4448.

57. Joosten LA, et al. Inflammatory arthritis in caspase 1 gene-deficient mice: contribution of proteinase 3 to caspase 1 -independent production of bioactive interleukin-1B. Arthritis Rheum. 2009;60(12):3651-3662.

58. Schreiber A, Pham CT, Hu Y, Schneider W, Luft FC, Kettritz R. Neutrophil serine proteases promote IL-1beta generation and injury in necrotizing crescentic glomerulonephritis. J Am Soc Nephrol. 2012;23(3):470-482.

59. Dinarello CA. Keep up the heat on IL-1. Blood. 2012;120(13):2538-2539.

60. Berda-Haddad Y, et al. Sterile inflammation of endothelial cell-derived apoptotic bodies is mediated by interleukin-1 $\alpha$. Proc Natl Acad Sci U S A. 2011;108(51):20684-20689.

61. Christenson K, Björkman L, Karlsson A, Bylund $\mathrm{J}$. Regulation of neutrophil apoptosis differs after in vivo transmigration to skin chambers and synovial fluid: a role for inflammasomedependent interleukin-1 $\beta$ release. J Innate Immun. 2013;5(4):377-388.

62. Teichmann LL, Schenten D, Medzhitov R, Kashgarian M, Shlomchik MJ. Signals via the adaptor MyD88 in B cells and DCs make distinct and synergistic contributions to immune activation and tissue damage in lupus. Immunity. 2013;38(3):528-540.

63. McInnis EA, et al. Dysregulation of autoantigen genes in ANCA-associated vasculitis involves 
alternative transcripts and new protein synthesis. J Am Soc Nephrol. 2015;26(2):390-399.

64. Freeley SJ, Coughlan AM, Popat RJ, DunnWalters DK, Robson MG. Granulocyte colony stimulating factor exacerbates antineutrophil cytoplasmic antibody vasculitis. Ann Rheum Dis. 2013;72(6):1053-1058.

65. Richter AG, Perkins GD, Chavda A, Sapey E, Harper L, Thickett DR. Neutrophil chemotaxis in granulomatosis with polyangiitis (Wegener's) and idiopathic pulmonary fibrosis. Eur Respir J. 2011;38(5):1081-1088.

66. Gerber EE, et al. Integrin-modulating therapy prevents fibrosis and autoimmunity in mouse models of scleroderma. Nature. 2013;503(7474):126-130.

67. Rimbert M, et al. Decreased numbers of blood dendritic cells and defective function of regulatory $\mathrm{T}$ cells in antineutrophil cytoplasmic antibody-associated vasculitis. PLoS One. 2011;6(4):e18734.

68. Csernok E, Szymkowiak CH, Mistry N, Daha MR, Gross WL, Kekow J. Transforming growth factorbeta (TGF- $\beta$ ) expression and interaction with proteinase 3 (PR3) in anti-neutrophil cytoplasmic antibody (ANCA)-associated vasculitis. Clin Exp Immunol.1996;105(1):104-111.

69. Tan C, Gery I. The unique features of Th9 cells and their products. Crit Rev Immunol. 2012;32(1):1-10.
70. Abdulahad WH, van der Geld YM, Stegeman CA, Kallenberg CG. Persistent expansion of $\mathrm{CD}^{+}$ effector memory T cells in Wegener's granulomatosis. Kidney Int. 2006;70(5):938-947.

71. Lamprecht P. L20. Memory T-cells in vasculitis. Presse Med. 2013;42(4 pt 2):560-563.

72. Wilde B, et al. Th17 expansion in granulomatosis with polyangiitis (Wegener's): the role of disease activity, immune regulation and therapy. Arthritis Res Ther. 2012;14(5):R227.

73. Abdulahad WH, Stegeman CA, Limburg PC, Kallenberg CG. Skewed distribution of Th17 lymphocytes in patients with Wegener's granulomatosis in remission. Arthritis Rheum. 2008;58(7):2196-2205.

74. Fagin U, et al. Distinct proteinase 3-induced cytokine patterns in Wegener s granulomatosis, Churg-Strauss syndrome, and healthy controls. Clin Exp Rheum. 2011; 29(1 suppl 64):S57-S62.

75. Velden J, et al. Renal IL-17 expression in human ANCA-associated glomerulonephritis. Am J Physiol. 2012;302(12):F1663-F1673.

76. Tamarozzi F, Wright HL, Thomas HB, Edwards SW, Taylor MJ. A lack of confirmation with alternative assays questions the validity of IL-17A expression in human neutrophils using immunohistochemistry. Immunol Lett. 2014;162(2 pt B):194-198.

77. Wilde $\mathrm{B}$, et al. Th17 expansion in granulomatosis with polyangiitis (Wegener's): the role of disease activity, immune regulation and therapy. Arthritis Res Ther. 2012;14(5):R227.

78. Abdulahad WH, et al. Increased frequency of circulating IL-21 producing Th-cells in patients with granulomatosis with polyangiitis (GPA). Arthritis Res Ther. 2013;15(3):R70.

79. Mills CD, Ley K. M1 and M2 macrophages: the chicken and the egg of immunity. J Innate Immun. 2014;6(6):716-726.

80. Witko-Sarsat V, et al. Proliferating cell nuclear antigen acts as a cytoplasmic platform controlling human neutrophil survival. J Exp Med. 2010;207(12):2631-2645.

81. Jennette JC, et al. 2012 revised International Chapel Hill Consensus Conference Nomenclature of Vasculitides. Arthritis Rheum. 2013;65(1):1-11.

82. Luqmani RA, et al. Birmingham Vasculitis Activity Score (BVAS) in systemic necrotizing vasculitis. QJM. 1994;87(11):671-678.

83. Hellmich B, et al. EULAR recommendations for conducting clinical studies and/or clinical trials in systemic vasculitis: focus on anti-neutrophil cytoplasm antibody-associated vasculitis. Ann Rheum Dis. 2007;66(5):605-617.

84. Watts R, et al. Development and validation of a consensus methodology for the classification of the ANCA-associated vasculitides and polyarteritis nodosa for epidemiological studies. Ann Rheum Dis. 2007;66(2):222-227. 